\title{
Holographic optical metasurfaces: a review of current progress
}

\section{Citation}

Genevet, Patrice, and Federico Capasso. 2015. "Holographic Optical Metasurfaces: A Review of Current Progress." Reports on Progress in Physics 78 (2): 24401. https:// doi.org/10.1088/0034-4885/78/2/024401.

\section{Permanent link}

http://nrs.harvard.edu/urn-3:HUL.InstRepos:41371376

\section{Terms of Use}

This article was downloaded from Harvard University's DASH repository, WARNING: This file should NOT have been available for downloading from Harvard University's DASH repository.

\section{Share Your Story}

The Harvard community has made this article openly available.

Please share how this access benefits you. Submit a story.

\section{Accessibility}




\title{
Report on Progress
}

\section{Holographic optical metasurfaces: a review of current progress}

\author{
Patrice Genevet $^{1,2}$ and Federico Capasso ${ }^{1}$ \\ ${ }^{1}$ School of Engineering and Applied Sciences, Harvard University, Cambridge, MA 02138, USA \\ 2 Singapore Institute of Manufacturing Technology, 71 Nanyang Drive, Singapore 638075, Singapore \\ E-mail: genevetp@simtech.a-star.edu.sg and Capasso@seas.harvard.edu
}

Received 18 June 2014, revised 12 October 2014

Accepted for publication 16 October 2014

Published 22 January 2015

Invited by Masud Mansuripur

\begin{abstract}
In this article, we review recent developments in the field of surface electromagnetic wave holography. The holography principle is used as a tool to solve an inverse engineering problem consisting of designing novel plasmonic interfaces to excite either surface waves or free-space beams with any desirable field distributions. Leveraging on the new nanotechnologies to carve subwavelength features within the large diffracting apertures of conventional holograms, it is now possible to create binary holographic interfaces to shape both amplitude phase and polarization of light. The ability of the new generation of ultrathin and compact holographic optical devices to fully address light properties could find widespread applications in photonics.
\end{abstract}

Keywords: metasurfaces, holography, surface plasmon polaritons, detour phase holograms

(Some figures may appear in colour only in the online journal)

\section{Introduction to surface electromagnetic wave holography}

Plasmonics, the study of the coupling between electromagnetic fields and electronic oscillations at metal-dielectric interfaces, has emerged as one of the most rapidly growing fields of optical physics (Maier 2007, Han and Bozhevolnyi 2013). Plasmonics has made it possible to move optics to the sub-wavelength scale, opening up a range of new opportunities in science and technology for communication, sensing, and imaging (Barnes et al 2003, Schuller et al 2010). However, for this vision to bear fruits, new components that can act as optical interconnects between nanoscale plasmonic volumes and free-space optical elements are still needed. In this review, we describe the advances in designing such components using a holographic approach. Holography, developed first in 1947 by Denis Gabor (Gabor 1948), is originally an imaging technique that consists of scattering an incident coherent source from an interference pattern (the hologram) such that the scattered fields reconstructs the $3 \mathrm{D}$ features of an object. Following the pioneering work of Gabor, the principle of holography was applied to free-space optical beams with the advent of the laser and later extended to surface waves by Cowan in 1972 (Cowan 1972). In the latter work, holograms were generated using surface waves as reference beams. Subsequent papers used the collective excitation of surface plasmon polaritons (SPPs), i.e. charge density waves propagating at the interface between metal and dielectrics (Shalaev and Kawata 2007), to create and record holograms (Maruo et al 1997, Wang et al 2001, Ozaki et al 2011).

We start our discussion by considering that in the dielectric half-space, the component of the surface plasmon polariton electric field normal to the metal-dielectric interface $E_{z}$ obeys Helmholtz's equation:

$$
\nabla^{2} E_{z}+\varepsilon_{d} k_{0}^{2} E_{z}=0
$$

The surface plasmon propagation constant $k_{\text {spp }}$ satisfies $k_{\text {spp }}^{2}=k_{x}^{2}+k_{y}^{2}=\alpha^{2}+\varepsilon_{d} k_{0}^{2}=k_{0}^{2} \varepsilon_{d} \varepsilon_{m} / \varepsilon_{d}+\varepsilon_{m}$ where $k_{0}$ is the free space propagation constant, $-\alpha$ is the inverse of the decay 
length of the electric field in the dielectric, $\varepsilon_{d}$ and $\varepsilon_{m}$ are the permittivity of the dielectric and the metal, respectively. In these latter works on surface waves holography, the interference between the light scattered by the object and the reference SPP beam produce a phase grating onto the photographic film placed in contact with the metallic surface. The information encoded in the film can then be reconstructed by sending a readout SPP beam propagating at the interface between the metal and the photographic film, see illustration in figure 1.

Inspired by some of these early works, a new class of interfaces that tremendously simplifies the problem of recording the hologram was proposed. Assuming a given reference and an object beam, it is not necessary to use the photographic layers previously used in holography; instead one can directly transfer computed holograms on films using standard lithography techniques (Brown and Lohmann 1969). In this review, we discuss how nanophotonics can advance the field of holography, introducing new techniques which are suitable for designing a new class of optical components for controlling amplitude, phase and eventually polarization of holographic objects. These techniques can alternatively be used to excite interesting surface waves for various applications in photonics. It consists of patterning a metallic film, the one that supports SPP propagation, with grooves or scatterers following an interferometric pattern, i.e. a holographic design (Chen et al 2011, Genevet et al 2012, Dolev et al 2012). SPPs propagating towards the hologram scatter from the corrugations and decouple to generate a free-space wavefront with predefined amplitude and phase distribution. Holographic interfaces can also be designed to launch any bounded SPP modes using free-space excitations. In section 2 of this review, we will discuss how to design plasmonic holograms. We will introduce a simple, but useful, phenomenological model for calculating the farfield radiation of SPP scattered at holographically structured interfaces (Tetienne et al 2011). In section 3, we will discuss the application of holographic couplers to surface plasmon polaritons and we will show that the holographic approach can be applied for launching SPPs from beams incident with non uniform spatial phase and amplitude distributions. One of the practical implementations of these holographic couplers is to achieve selective detection of the orbital angular momentum (OAM) of light. In section 4, we will report a new type of holographic interface, which after being designed for a given wavelength, is able to manipulate the three fundamental properties of light (phase, amplitude and polarization) over a broad wavelength range. This design strategy relies on replacing the large openings of conventional holograms by arrays of sub-wavelength apertures, oriented to locally select a particular state of polarization. The resulting optical element can therefore be viewed as the superposition of two independent structures with very different length scales, i.e. a hologram with each of its apertures filled with nanoscale openings to transmit only a desired state of polarization. As an implementation, we present a nano-structured holographic plate to generate radially polarized optical beams from circularly polarized incident light. We will show that it operates over a broad range of wavelengths.

\section{Holographic plasmonic couplers}

The new class of plasmonic holographic gratings discussed in this section can generate, for example, multiple free-space beams in arbitrary directions from a point source of surface waves. We will introduce the concept of surface wave holography and derive a phenomenological model which accurately predicts the far-field, in amplitude, phase and polarization of a surface wave scattered by a distribution of grooves disposed following a holographic pattern.

\subsection{Gratings: examples of plasmonic holograms}

The general problem of coupling a surface plasmon polariton plane wave to a free-space plane wave is usually addressed using one-dimensional (1D) gratings (Garcia-Vidal et al 2003, Martín-Moreno et al 2003, Yu et al 2005, Kim et al 2007, Yu et al 2008) (figure 2(a)). The latter consists of periodic corrugations at an interface with a periodicity $\Gamma$ to fulfill the phase matching condition:

$$
k_{i}+k_{G}=k_{\mathrm{spp}}
$$

with $k_{i}=k_{0} \sin \theta, k_{G}=2 \pi / \Gamma$ and $k_{\text {spp }}=2 \pi / \lambda_{\text {spp}}$. In order to satisfy this momentum conservation, the locations of grooves, which encode the phase and amplitude information of the scattered light, coincide with the lines of maximum of interference between a SPP reference wave and the scattered free-space plane wave (figure $2(b)$ ). 1D gratings can therefore be viewed as a holographic coupler since the scattering lines are positioned where we would find the maximum of interference between the SPP reference beam and the real object. As an example, a normally incident beam interferes with an SPP propagating on a metallic surface to produce a cosine intensity interferogram with periodicity equal to the SPP wavelength. By etching apertures along the maximum of those lines, we obtain a coupler (grating coupler) which couple normally incident beam into SPPs (figure 2(b)).

Two-dimensional (2D) gratings obtained by a rotation of a second-order 1D grating (also named bull's-eye structures) were also used to generate a collimated beam from a pointlike source of surface waves, or reversely to focus incident light into a nanoscale-size 'hot spot' (Lezec et al 2002, Ishi et al 2005, Liu et al 2005, Lee et al 2010). Such structures, which are still 1D in a cylindrical coordinate system, can also be viewed as holographic couplers with azimuthal symmetry.

\subsection{Holography with surfaces waves: a dipolar model}

\subsubsection{An inverse scattering approach.}

We would like to start this discussion by deriving an analytical expression of the holographic patterns. In the process of recording a conventional hologram, one usually needs to shine two coherent beams which interfere and write the interferogram on a recording media. One of the two coherent beams is traditionally called the object beam. In this discussion, we consider an object beam in the far-field $E_{\mathrm{ff}}=\mathrm{FT}\left\{E_{\mathrm{obj}}(x, y)\right\}$ where FT denotes the Fourier transform. The phase and amplitude distribution of the Fourier transform of the object in the metallic plane $(z=0)$ can be written as $E_{\text {obj }}(x, y)=A_{\text {obj }}(x, y) \mathrm{e}^{\mathrm{i} \psi_{\text {obj }}(x, y)}$ 

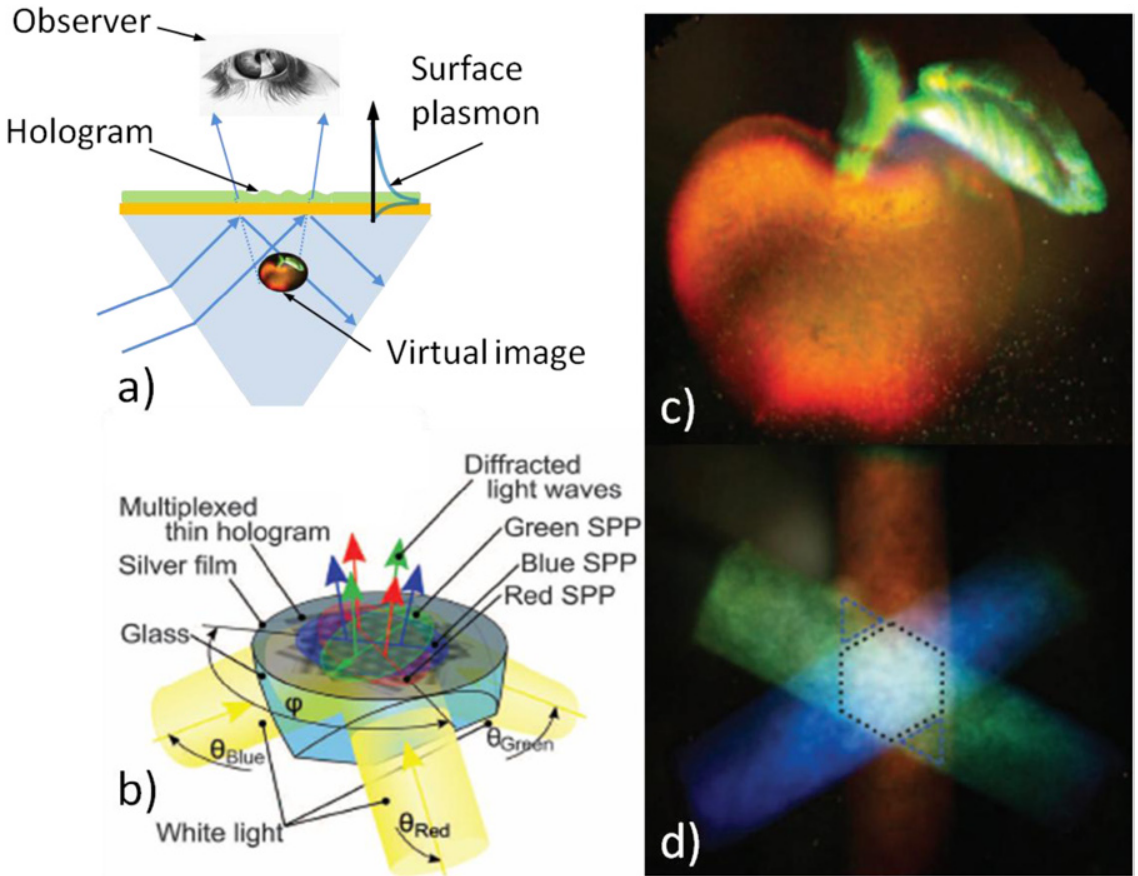

Figure 1. (a) Illustration of the principle of surface electromagnetic wave holography with a holographic film deposited at the interface gold/air. (b) Reconstruction of a color object through SPP hologram. The hologram is illuminated simultaneously with white light in three directions at different angles. $(c)$ 3D holographic image of an apple. (d) Color bar recorded three times with red, green, and blue by rotating the bar by $120^{\circ}$ for each color. In the center, where the three colors overlap, a hexagonal area is reconstructed as white, whereas yellow triangles are reconstructed where red and green overlap. Figures reproduced and adapted with permission from Ozaki et al (2011).

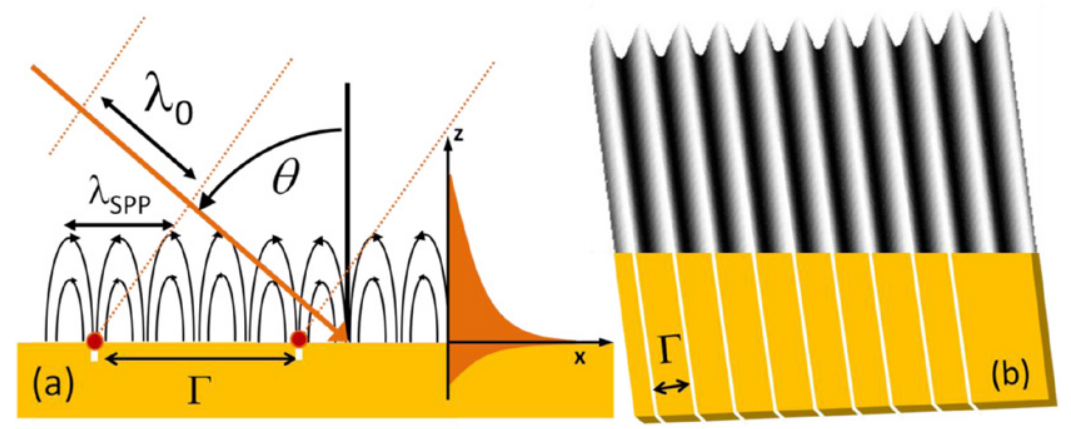

Figure 2. (a) Gratings, made of periodically arranged apertures in metallic films, are used to illustrate the concept of holographic couplers. The periodic corrugations with a periodicity $\Gamma$ fulfill the wavevector compensation parallel to the interface: $k_{i}+k_{G}=k_{\text {spp }}$, with $k_{i}=k_{0} \sin \theta$, $k_{G}=2 \pi / \Gamma$ and $k_{\text {spp }}=2 \pi / \lambda_{\text {spp. }}$. A plane wave incident on a grating at the correct angle and at the correct wavelength launches a plane wave of SPPs. (b) Interference between a SPP plane wave and a reference beam incident onto the metallic film at an angle $\theta$ produces an interferogram with lines of maximum intensity separated by a period $\Gamma$, i.e. separated by the same spatial interval than the corresponding grating coupler in $(a)$.

(Dolev et al 2012). The second beam, a propagating surface plasmon polariton beam, is called the reference beam denoted by $E_{\text {ref }}(x, y)=A_{\text {ref }} \mathrm{e}^{\mathrm{i} \phi_{\text {ref }}(x, y)}$. Both interfere to produce an interferogram with an intensity distribution following:

$$
\begin{aligned}
& I(x, y)=\left[E_{\mathrm{obj}}+E_{\mathrm{ref}}\right] \cdot\left[E_{\mathrm{obj}}^{*}+E_{\mathrm{ref}}^{*}\right]=A_{\mathrm{obj}}^{2}+A_{\mathrm{ref}}^{2}+E_{\mathrm{obj}}^{*} \\
& E_{\mathrm{ref}}+E_{\mathrm{obj}} E_{\mathrm{ref}}^{*}=A_{\mathrm{obj}}^{2}+A_{\mathrm{ref}}^{2}+2 * A_{\mathrm{obj}} A_{\mathrm{ref}} \\
& \cos \left(\psi_{\mathrm{obj}}(x, y)-\phi_{\mathrm{ref}}(x, y)+c_{1}\right)
\end{aligned}
$$

High intensity lines, which according to equation (3) follow:

$$
\psi_{\mathrm{obj}}-\phi_{\mathrm{ref}}+c_{1}-2 m \pi=0
$$

where $m$ is an integer, define the location on the metal film where one needs to etch the metal to create scattering apertures. In this approach, the role of the phase retardation introduced by the scattering event, for example when the reference beam propagates through a corrugation, is taken into account by the phase shift $c_{1}$ in equation 4 . If the grooves are relatively small and shallow compared to the wavelength, i.e. non-resonant apertures, this phase shift can be neglected (Chen et al 2011). Given a desired object ${ }^{3}$ and a reference SPP beam,

\footnotetext{
${ }^{3}$ In fact, the object's far field Fourier transform. To simplify the discussion, we consider the field distribution of the object after propagation towards the interface.
} 


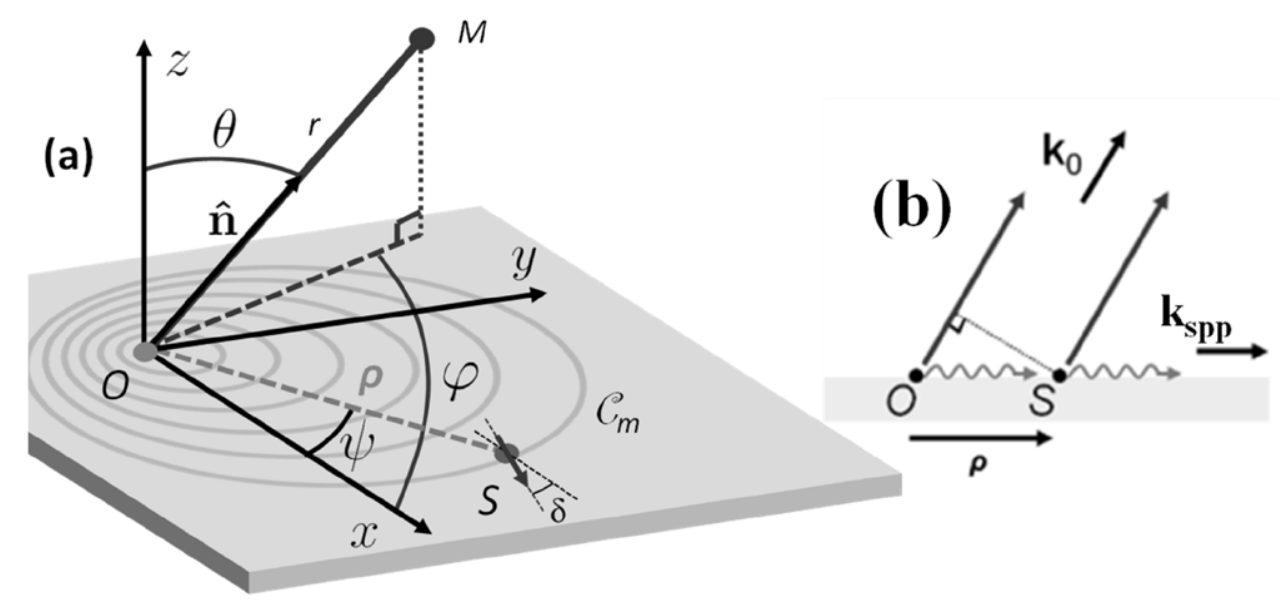

Figure 3. (a) Schematic of a holographic coupler. Grooves (noted $C_{m}$ ) are etched on the metal film at locations where the surface plasmon polaritons and the object beam would interfere constructively. A SPP beam propagating from the center O will eventually be scattered in the far field to recreate the object beam. (b) Interference between the field radiated by the source in $\mathrm{O}$ and the field scattered by the scatterers $\mathrm{S}$. Reproduced from Blanchard et al (2011).

one can compute the intensity distribution and transfer the resulting pattern onto a metallic film ${ }^{4}$. After designing the coupler, we can produce the desired object beam by sending a so-called reading beam. The latter is therefore a SPP beam which propagate on a corrugated interface and scatters at each groove to interfere in the far-field, forming the object beam $E_{\mathrm{ff}}$. The essential physical phenomenon involved in this process is the interference between the emissions of multiple scattering grooves ${ }^{5}$ distributed along the surface.

\subsubsection{Far-field scattering from a holographic coupler.}

In the following, we discuss a phenomenological model to calculate the far-field radiation pattern of any 2D pattern designed by following the holographic design approach. We approximate the field scattered by the apertures as that of an ensemble of electric dipoles distributed on the surface (Tetienne et al 2011). Ray tracing models have already been used to describe 2D focusing holographic gratings (Heitmann 1981). The model briefly described here, and in detail in Tetienne et al (2011), is even more versatile: by using a 'microscopic' description of the apertures, that is, by replacing the apertures in a metal film by an assembly of dipolar emitters, we can achieve an accurate modeling of complex structures with finite size and local parameters such as illumination intensity, scattering efficiency, losses, and so on.

Suppose that one wants to design a coupler which would scatter surface waves departing from a point source $O$ into a free space Gaussian beam (or reciprocally from a Gaussian incident free space beam into surface waves focused in $O$ ). To design this coupler, we considered the interference pattern of the surface waves originating from a focal point $O$ with the desired beam. As sketched in figure 2(b), following the fringes with maximum of the interference pattern, one constructs an ensemble of lines $C_{m}$ which represent the equi-phase location of the surface waves and the free space beam. During the

\footnotetext{
${ }^{4}$ Due to the transverse magnetic (TM) character of SPP modes, only the inplane component of the SPP field can interfere with the object beam, which implies that about $10 \%$ of the SPP energy participates in the process.

${ }^{5}$ The lines could be replaced by holes, particles, grooves or even ridges.
}

propagation on the metallic interface, SPPs are scattered by the corrugation disposed along the metal-air interface and the effect of these corrugations can be modeled as an ensemble of point scatterers $S$ lying on the lines $C_{m}$ in the $x-y$-plane (figure $3(a)$ ). The point source $\mathrm{O}$ can often be modeled as a point dipole whose polarization imposes a particular angular distribution on the launched SPPs (Yu et al 2010). The lines $c_{m}$ describe the grooves of a generic $2 \mathrm{D}$ grating. We assume that each point $S$, with polar coordinates $(\rho, \Psi)$, scatters a portion of the SPPs out of the plane, while the other portion keeps propagating along the surface. The radiation pattern of this system is obtained by summing over all scattered fields.

We assume that the field $\mathrm{d} E_{S}(r)$ scattered by an infinitesimal portion of $C_{m}$ around $S$, subtending an angle $\mathrm{d} \psi$, can be modelled by that of an electric dipole located at $S$, and having an amplitude proportional to the amplitude of the SPP at that point, modulated by a scattering efficiency. The SPP complex amplitude at $S$ can be expressed as $a_{S}=f_{1}(\rho) f_{2}(\psi) \mathrm{e}^{\mathrm{i} k_{\mathrm{spp}} \rho}$, where $f_{1}(\rho)$ expresses the decay of the SPPs as they propagate away from the source (due to diffraction, scattering, and absorption losses), and $f_{2}(\psi)$ is the angular distribution of the SPPs launched at the point source. $k_{\mathrm{spp}}$ is the SPP wavevector. For a point source polarized at an angle $\psi=\psi_{s}, f_{2}(\psi)$ can be expressed as $f_{2}(\psi)=\cos \left(\psi-\psi_{s}\right)$ (Yu et al 2010). As the lines of scatterers represent grooves, the orientation of the dipole is taken in the $x-y$-plane, normal to the groove. If $\widehat{\boldsymbol{\delta}}$ is a unit vector which defines the orientation of the dipole, $\delta$ being the angle formed with the $x$-axis, one can write:

$\mathrm{d} E_{S}(r) \propto a_{S} f_{3}(\delta-\psi) \frac{\mathrm{e}^{\mathrm{i}\left(k_{0} \hat{\mathbf{n}} .(\mathbf{r}-\rho)+c 1\right)}}{\|r-\rho\|}[(\hat{\mathbf{n}} \times \hat{\boldsymbol{\delta}}) \times \hat{\mathbf{n}}] \mathrm{d} \psi$

where $\boldsymbol{n}$ is the unit vector in the direction $O M$. The source is assumed monochromatic, with a free-space wavelength $\lambda_{0}=2 \pi / k_{0}$. We neglect the reflections of SPPs at the grooves, which is a valid approximation except in the case of constructive interference of the small reflections from each groove, which sets in when the grating period is about $m . \lambda_{\mathrm{spp}} / 2$, where $m=1,2,3 \ldots$. 
The groove scattering efficiency at $S$ is denoted by $f_{3}(\delta-\psi)$. It depends on the direction of the incident SPPs relative to the groove, and on the groove depth and width. The phase shift introduced by the scattering event is $c_{1}$. We emphasize that the input parameters of our model $\left(f_{1}, f_{3}\right.$ and $\left.c_{1}\right)$, specific to a given groove geometry and working wavelength, can be readily determined through simple considerations and $2 \mathrm{D}$ simulations.

Once these parameters are evaluated, it is possible to study the response of all sorts of holographically designed couplers by simply changing the equation of the curves $C_{m}$, predicting not only the amplitude pattern but also the phase pattern of the radiated light. The expression of the total field is obtained by summing over the continuous distribution of scatterers $S$ on the line $C_{m}$, and over $N$ lines. The radiated power density in the far-field is proportional to the angle-dependant component of $\left|E_{\text {obj }}(r)\right|^{2}$ :

$$
\left.D_{\text {obj }}(\theta, \varphi) \propto\left|\sum_{m=1}^{N} \int_{0}^{2 \pi} \mathrm{e}^{\mathrm{i} \Delta \phi} f_{1} f_{2} f_{3} \hat{\mathrm{e}}(\theta, \varphi, \delta) \mathrm{d} \psi\right|\right|^{2}
$$

where $\hat{\mathbf{e}}(\theta, \varphi, \delta)=\cos (\theta) \cos (\delta-\varphi) \hat{\boldsymbol{\theta}}+\sin (\delta-\varphi) \hat{\boldsymbol{\phi}}$. Here, $\Delta \phi$ is the phase difference between the field $E_{\mathrm{obj}}$ scattered by $S$ and the field $E_{\text {ref }}$ directly emitted from the source $O$, both evaluated at point $M$ with spherical coordinates $(r, \theta, \varphi)$ in the far-field $(r \gg d \gg \lambda$ ), the grating dimensions being of order of $d$. It is given by:

$$
\Delta \phi=k_{\mathrm{SPP}} \widehat{\boldsymbol{m}} \cdot \boldsymbol{\rho}-k_{0} \hat{\mathbf{n}} \cdot \boldsymbol{\rho}+c_{1}
$$

where $\widehat{\boldsymbol{m}}$ is the unit vector in the direction OS. We use this model to devise a general class of holographic couplers such as plasmonic lenses that collimate light originating from a single point source and direct it into one or several free-space beams propagating along arbitrary off-axis directions, or SPP couplers with vortex beams to redirect free space vortices into focused SPPs, thus enabling flexible interfacing of plasmonic devices with complex optical systems. The model developed here enables us to calculate the far-field radiation pattern of any $2 \mathrm{D}$ scattering interface, once the equation of the curves $C_{m}$ is known.

\section{Applications of surface holograms}

\subsection{Surface holograms to collimate the emission of lasers}

To demonstrate this concept, we are interested in a particular coupler that would scatter SPPs originating from a point source into a collimated free-space beam of arbitrary direction $(\theta, \varphi)$. We obtain the equation of the curves $C_{m}$ for such a grating by following the holographic approach discussed in the previous section. The position of the grooves is therefore given by equation (4). Introducing the SPP effective index $n_{\text {spp }}$, we obtain the equation that yields the position of the scatterers:

$$
\rho=\rho_{m}(\psi)=\lambda_{0} \frac{m-c_{1} / 2 \pi}{n_{\mathrm{spp}}-\sin (\theta) / \cos (\psi-\varphi)}
$$

This equation states that the scatterers must be located on the curves $C_{m}$ defined by the polar equations $\rho=\rho_{m}(\psi)$ for all emitted field to constructively interfere in the direction $(\theta, \varphi)$. The curves $C_{m}$ are ellipses with the origin $O$ at one of their foci.

The far-field radiated by such elliptical system of grooves has been first calculated and then measured by fabricating the holograms directly on the gold-coated facet of mid-infrared quantum cascade lasers (QCLs) emitting at $\lambda=8.06 \mu \mathrm{m}$, using the process described in (Yu et al 2008). The results are presented in figure 4 and more details can be found in Tetienne et al (2011). In this device, the point-like source of SPPs is a subwavelength aperture opened in the metal film (figures 4(a) and $(b))$. The light in the laser waveguide impinges on the back side of the aperture, launching SWs on the grating-air interface, along with some directly transmitted light. This data demonstrates that 2D holographic couplers can transform a point source of SPPs located at the focal point into one or multiple collimated free-space beams in a tailorable direction, i.e. it acts as planar plasmonic lens. Since this model is vectorial, one can also extract the state of polarization of the emitted light see details in Tetienne et al (2011).

\subsection{Detection of orbital angular momentum of light with plasmonic photodiodes}

In the following, we shall discuss the potential applications of the holographic design to launch various sort of plasmonic surface beams. As an example, we integrated plasmonic couplers into commercial silicon photodiodes and achieve selective detection of the orbital angular momentum (OAM) of light (Genevet et al 2012). Light with OAM, also called vortex beams, have a doughnut-like transverse intensity profile and carry an OAM of $\hbar L_{i}$ per photon, where $L_{i}$ is the number of twists of the wavefront along a distance equal to the wavelength $^{6}$ and $\hbar$ is Planck's constant. OAM states are orthogonal and beams with different OAM can propagate collinearly while carrying a quantum number of any integer value. These intriguing properties make light with OAM appealing for applications in microscopy, optical trapping and optical communication (Leach et al 2002, Hell 2007, Padgett and Bowman 2011, Torres and Torner 2011). Since the topological charge of an optical vortex beam can be annihilated by interacting with another beam carrying an opposite vorticity, they have been considered as the optical analogue of superfluid vortices (Coullet et al 1989, Genevet et al 2010). To fully exploit the potential of OAM for applications, several techniques have been developed to selectively detect OAM (He et al 1995, Golub et al 1996, Mair et al 2001, Lavery et al 2012). All of these free-space methods require bulky systems comprising multiple components which are usually not cost effective and are difficult to implement. Recently, integrated photonic waveguide plates have been proposed to sort the OAM on-chip by sampling the wavefront of vortex beams using vertical gratings that couple light into phased-array waveguides ( $\mathrm{Su}$ et al 2012).

By using the unique properties of SPPs, we can measure the OAM with conventional photodetectors. Unlike

\footnotetext{
${ }^{6}$ This quantity is also called the topological charge of the phase singularity.
} 

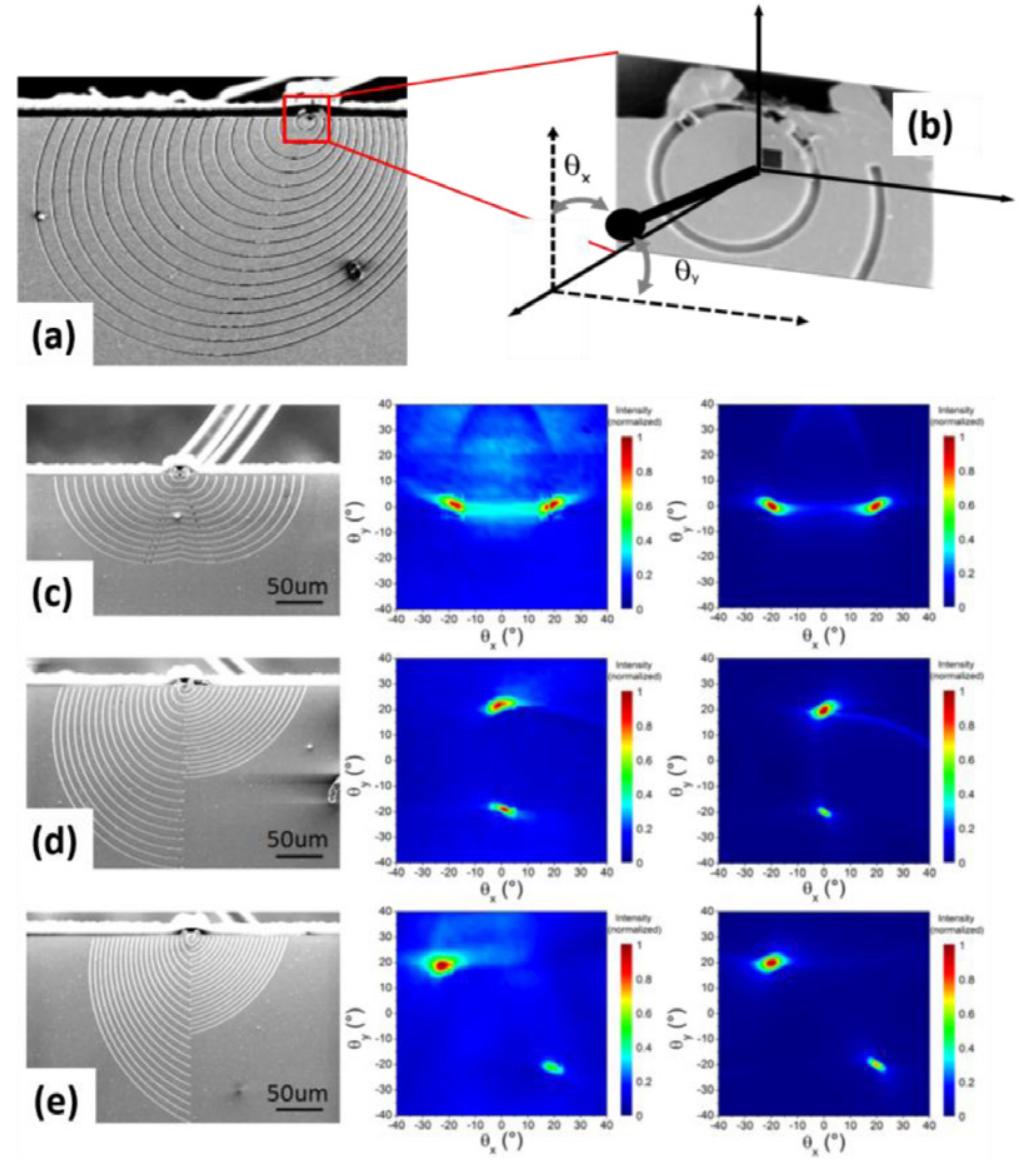

Figure 4. (a) is an example of a one-beam collimator. (b) Close-up image of a typical aperture and notation used to express the radiated far field. (c)-(e) are different plasmonic lenses designed to produce two beams at different angles, fabricated on the facet of quantum cascade lasers. For each structure, we show an SEM image (left column), and the measured (middle column) and calculated (using the model we develop above) (right column) far-field intensity (normalized to 1). The measured far-fields are obtained by scanning a mercury-cadmium-telluride detector, positioned 10-20 cm away from the device, over $\theta_{x}$ and $\theta_{y}$ with a resolution of 0.5 degrees where $\theta_{x}$ and $\theta_{y}$ are related to $(\theta, \varphi)$ following the diagram in $(d)$. Figures reproduced with permission from Tetienne et al (2011). ( ) IOP Publishing and Deutsche Physikalisene Gesellschaft.

plane waves and Gaussian beams, the phase profile of vortex beams is changing in the plane transverse to the direction of propagation, therefore making vortex beam to SPPs coupling process quite cumbersome. The previously discussed holographic design technique can solve this problem. Following the recipe exposed during the second section, we designed holographic couplers for incident vortex beam by computing the interferogram at the interface between converging SPP waves and free-space beams carrying OAM. More specifically, we are interested in designing a particular coupler that would scatter SPPs originating from a point source into a vortex object beam impinging on the metallic film at normal incidence. Vortex beams can be expressed at $z=0$ by $E_{\text {obj }}(x, y)=\boldsymbol{A}_{\text {obj }}(r, z=0) \mathrm{e}^{\mathrm{i} L_{i} \cdot \psi}$, where $\psi=\tan ^{-1}(x / y) ; A(r, z)$ is the transverse Gaussian beam profile; $r$ is the radial distance from the beam center and $z$ is the axial distance from the beam waist. The position of the grooves responsible for the coupling between the two beams is then given by:

$$
\rho=\rho_{m}(\psi)=\lambda_{0} \frac{L_{i} \cdot \frac{\psi}{2 \pi}-m}{n_{\mathrm{spp}}}
$$

where $n_{\mathrm{spp}}$ is the SPP effective index. We assume that the grooves are non-resonant, i.e. $c_{1}=0$, which is a reasonable approximation considering the size of the grooves of the fabricated devices. This equation states that the scatterers must be located on the curves $C_{m}$ defined by the polar equations $\rho=\rho_{m}(\psi)$ for all emitted fields to constructively interfere forming an optical vortex beam in the direction $(\theta=0, \varphi=0)$. According to the principle of reversibility of light, a vortex beam with a topological charge $L_{i}$ impinging at normal incidence on the structure defined by the equation (9) would launch a focusing beam of SPPs (see illustration in figure 5).

From the interference pattern, we extract the binary version of the hologram (figure $5(c)$ ) which will be transferred to the metallic film. In figure $5(d)$, we show a micrograph of a holographic interface fabricated by focused ion beam (FIB) milling. Our structures were designed to work for visible light at a wavelength of $633 \mathrm{~nm}$. As we mentioned, holographic couplers have potential for applications in various areas of integrated optics and by combining these metallic interfaces with a standard commercial photodiode, we demonstrated an integrated approach to sort the various incident OAM states of light. To do so, we patterned a silicon-based photodetector 

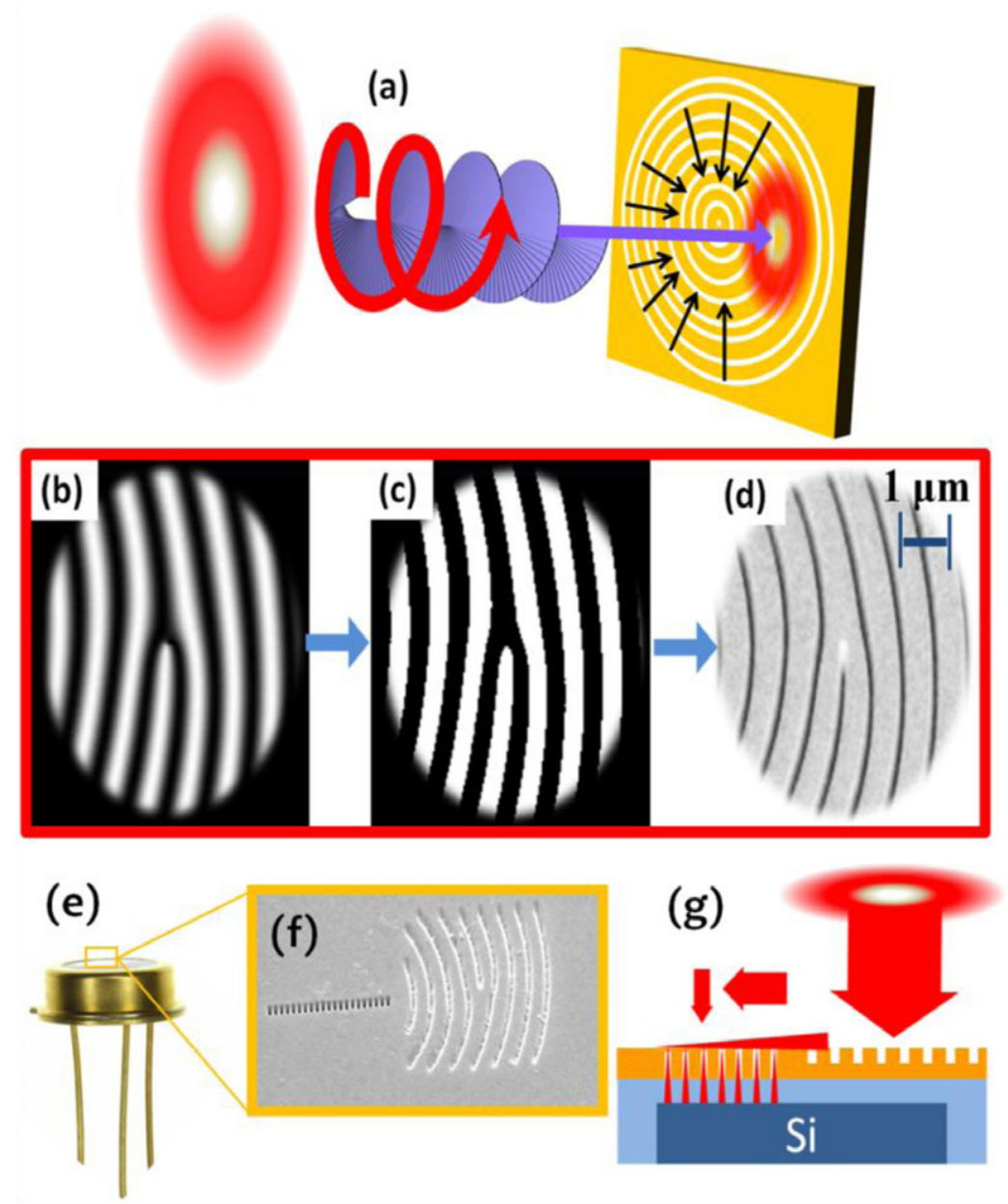

Figure 5. Interferometric design of the plasmonic couplers (a) Schematic explaining the approach used to design our holographic interfaces. Holographic couplers are designed by considering the interference between an incident beam with a complex wavefront, such as a vortex beam with a topological charge $L_{i}=-1$, impinging on the metal interface at normal incidence and a converging surface plasmon polariton (SPP) beam. (b) Computer generated interferogram. (c) Binary version of the interferogram in $(b)$. The bright lines represent the locations of equal phase (modulo $2 \pi$ ) of the two beams, where maximum constructive interference occurs. $(d)$ Scanning electron micrograph of a fabricated holographic interface where the grooves are placed at the equi-phase locations. (e) A photodetector with large active area is covered with metal on which we patterned an array of subwavelength hole with a subwavelength period $(f)$ and a holographic coupler. As the holes linear array pierces all the way through the gold film, the holes depth is equal to the metallic film thickness, their size is about $100 \mathrm{~nm}$ and the spacing is chosen as $200 \mathrm{~nm}$. The holographic coupler is designed to launch a focusing beam of SPP from an incident vortex beam $(\mathrm{g})$. The focused SPPs interact with the array of holes to funnel light through the gold film directly towards the active region of the detectors. Reproduced from Genevet et al (2012).

(Hamamatsu S2386-18K) with the holographic couplers discussed above. The photodiodes used for our experiments have very large active area (about $13 \mathrm{~mm}^{2}$ ) and exhibit a responsivity $\sim 200 \mathrm{~mA} \mathrm{~W}^{-1}$ at $\lambda=633 \mathrm{~nm}$. Figure 5(e) shows typical devices patterned with $L_{g}=1$ vortex hologram on its front window which were first coated with a $200 \mathrm{~nm}$ thick gold film using electron beam evaporation. An array of holes is placed at the correct distance from the hologram to allow for preferential transmission through the holes of the focused SPP generated by a vortex beam of specific OAM. Thus, it acts as a spatial filter to select only the focused plasmon fields. An incident beam with the correct OAM will be focused at the center of the device while beams with any other topological charges will be defocused away from the array of holes and hence will not be detected. To avoid direct coupling of light into the detector, we choose the distance between the holes to be much smaller than the SPP wavelength. The size of the holes is also sub-wavelength, to funnel light into the diode via extraordinary optical transmission (Ebbesen et al 1998, Lopez-Tejeira et al 2007, Weiner 2009).

The setup used to characterize the holographic OAM detectors consists sending a $\mathrm{TEM}_{00}$ laser mode of a linearly polarized $\mathrm{He}-\mathrm{Ne}$ laser, emitting at $633 \mathrm{~nm}$, previously incident on a programmable spatial lightwave modulator (Hamamatsu LCOS-SLM X 10468) to modify its OAM. A half wave plate is inserted along the beam path to control the polarization of the incident light onto the detector figure 6(a). Photocurrent is measured for two different holographic photodiodes, one patterned with a classical plasmonic lens $\left(L_{g}=0\right)$ that focuses a Gaussian beam (figure 6(b)) and the other with a plasmonic lens $\left(L_{g}=1\right)$ that focuses a vortex beam with $L_{i}=-1$ (figure $6(c)$ ) as a function of both 


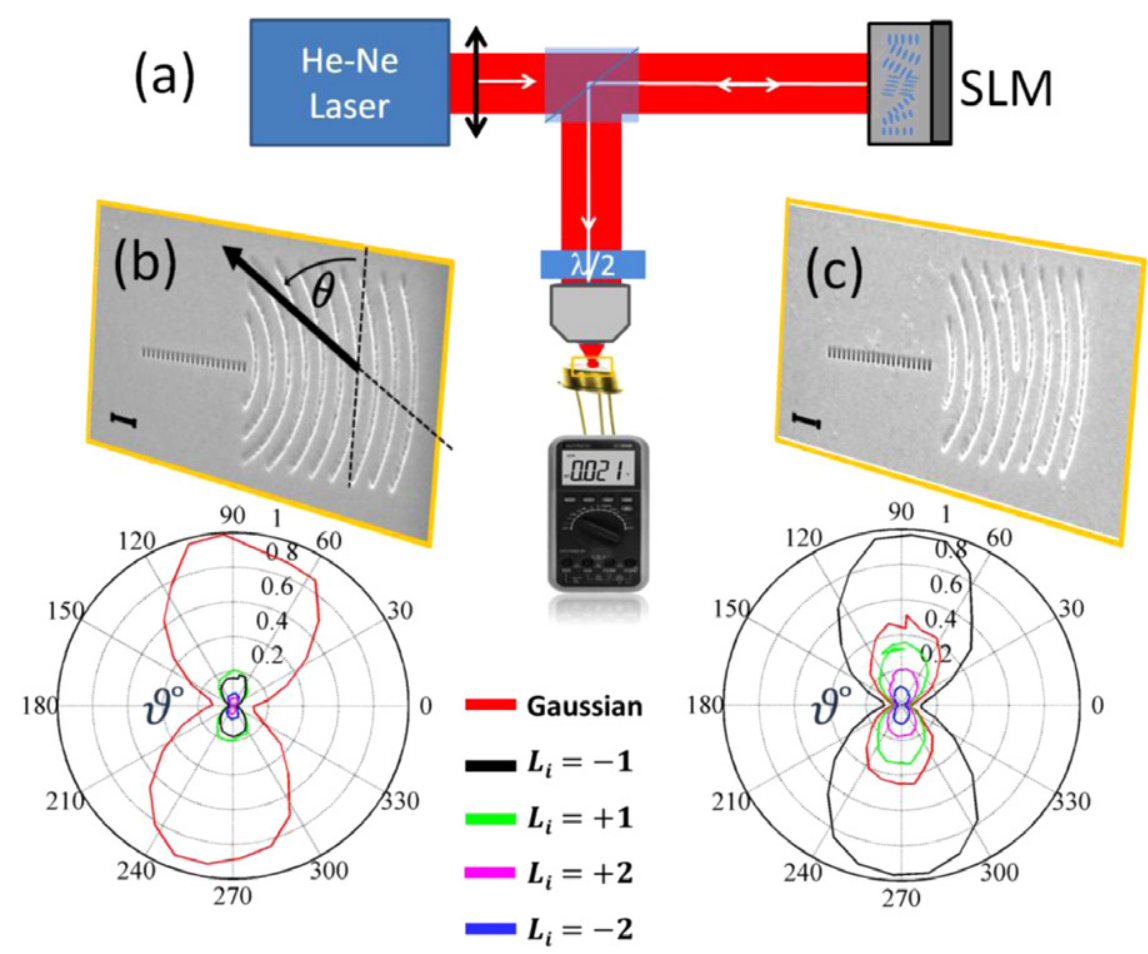

Figure 6. Experiment to detect the OAM with patterned photodiodes. (a) Schematic of the experiment. A spatial lightwave modulator (SLM) imparts to the incident laser beam a spiral-shaped wavefront of well-defined orbital angular momentum. A half-waveplate is used to control the incident polarization. The generated photocurrent is measured with a Keithley model 2400 ammeter. The figure also shows the electron micrographs (scale bar represents $1 \mu \mathrm{m})$ of two holograms patterned on top of the detectors: $(b)\left(L_{g}=0\right)$ is a conventional plasmonic lens that focuses a Gaussian beam with $L_{i}=0 .(c)\left(L_{\mathrm{g}}=1\right)$ is designed to focus a vortex beam with orbital angular momentum $L_{i}$ $=-1$ onto the array of holes. Reproduced from Genevet et al (2012).

polarization (the inset shows the orientation of the incident electric field with respect to the grooves) and the incident OAM (each color denotes a vortex beam with a different topological charge). The experimental results show that we can maintain a relatively good selectivity. In figure $6(b)$, the maximum photocurrent is observed for an incident Gaussian beam, i.e. when the incident beam matches the design of the hologram, and the signal decreases considerably (below $10 \%$ ) for beams with OAM. Conversely, figure 6(c) shows that the maximum photocurrent is measured for an incident vortex beam with $L_{i}=-1$. We varied the incident polarization and we confirmed that, for both photodiodes, the maximum signal is obtained for an incident polarization oriented normal to the grooves of the holographic interface, i.e. when the coupling to SPPs is maximized. The cross talk between different OAM channels is mostly due to the fact that the intensity along the central line of the propagating surface plasmon wave is not exactly zero when the incident topological charge is not the same as the desired one. Moreover, both in our experiments and simulations, OAM beams are created by converting a linearly polarized Gaussian TEM $_{00}$ into hypergeometric-Gaussian modes with non-zero OAMs. These modes, which have singular phase profiles, form an overcomplete but non-orthogonal set of modes and small cross talk is expected between modes with different OAMs (Karimi et al 2007). Additional misalignments during the experiments and imperfections in fabrication may contribute to increase the cross-talk signal which would eventually limit the performance of a communication system based on OAM (Gibson et al 2004, Wang et al 2012).

During the coupling process to the plasmons, the incident light carrying OAM is converted into an electromagnetic wave which carriers no OAM. During this process, angular momentum is conserved: assuming a vortex beam, angular momentum conservation requires $L_{i}+L_{g}=0$, where $L_{g}$ is provided by the holographic coupler to cancel $L_{i}$. Physically, the plasmonic lens with a fork-like dislocation fringe appearing at its center is designed to impart an opposite OAM to the incident vortex beam so that it can excite a surface wave with no vorticity, i.e. a focused SPP.

\subsection{Tailoring the surface electromagnetic field distribution of plasmonic beams with holographic couplers}

\subsubsection{Holographic launching of the $2 D$ non-diffracting plas- monic beams.}

Recent work demonstrated the existence of a new non-diffracting solution of the 2D Helmholtz equation: the cosine-Gauss beam (CGB) (Lin et al 2012). Its field profile is given by:

$E_{z}^{\mathrm{CGB}}=A \cdot f(x) \cdot \exp \left(j k_{x} x\right) \cos \left(k_{y} y\right) \exp \left(-\frac{y^{2}}{w_{0}^{2}}\right) \exp (\alpha z)$

where $w_{0}$ is the Gaussian waist, the term $\alpha$ in the exponential accounts for the evanescent decay of the field in the 


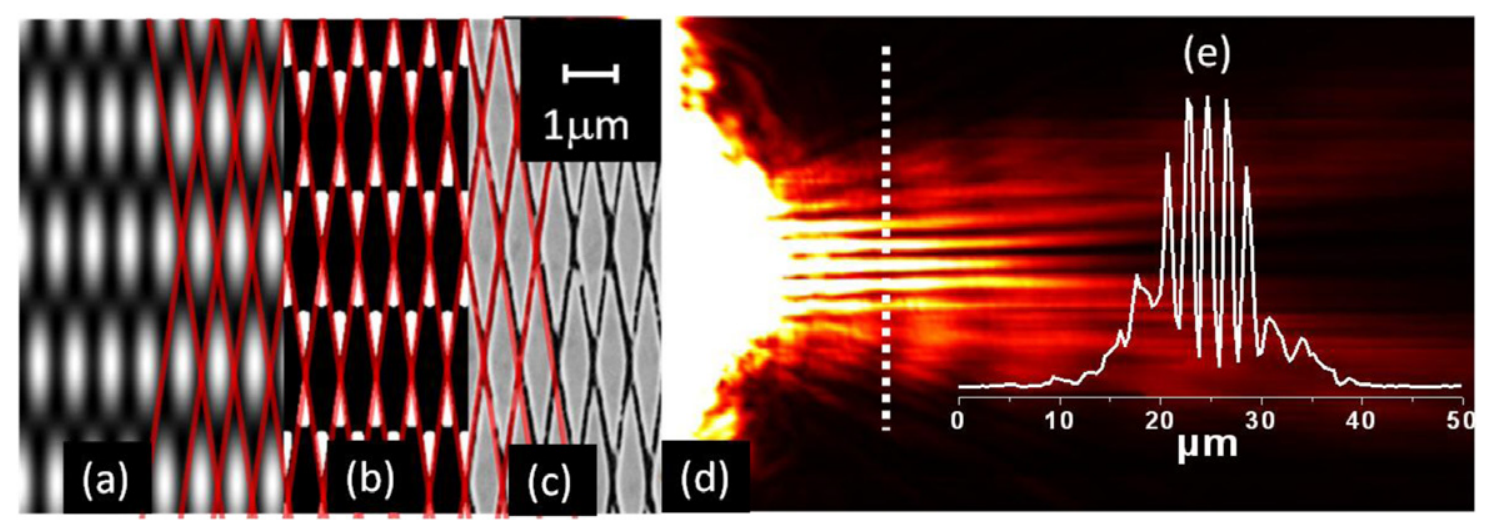

Figure 7. (a) Interferogram of normally incident plane wave with two SPP waves propagating at an angle $\theta_{\mathrm{CGB}}=20^{\circ} \mathrm{Deg}$. (b) Zerointensity lines in the interferogram. $(c)$ Digital implementation of the latter etched in metallic film. The red lines are used as a guide to the eyes to identify the zero-intensity lines in the hologram. $(d)$ Near-field scanning optical microscopy image representing the near field distribution of the cosine-Gauss beam. Due to its non-diffracting properties, the transverse field distribution $(e)$ does not spread during propagation.

dielectric, and $f(x)$ is described in (Lin et al 2012). As previously discussed in Lin et al (2012), Regan et al (2012), Garcia-Ortiz et al (2013) and Li et al (2013), CGBs are formed by the interference of two 2D plane waves with intersecting directions of propagation. The straight lines along which constructive interference occurs define the propagation direction of the CGB. Its propagation constant is given by $k_{x}^{\mathrm{CGB}}=k_{\mathrm{sp}} \cos \left(\theta_{\mathrm{CGB}}\right)$ where $\theta_{\mathrm{CGB}}$ is the half angle between the directions of propagation of the two plane wave components. By multiplying the two plasmonic plane waves by a Gaussian envelope of finite size, we demonstrated that the CGB remains non-diffracting in the paraxial approximation, while carrying finite amount of energy. While this envelope localizes the solution in a similar way as for Bessel Gauss beams (Gori et al 1987), it also introduces negligible diffraction effects which are unnoticed due to the relatively short SPP the propagation distance, i.e. the main narrow lobe of the CGB diffracts very slowly as a beam with the same transverse dimension as the Gaussian envelope. Note that this envelope is significantly larger than the narrow width of the main intensity lobe of the CGB, explaining its non-diffracting behavior. The CGB propagates in a straight line with a constant and controllable phase velocity, which can be modified by changing the half angle $\theta_{\mathrm{CGB}}$ between the two plane waves. To design a plasmonic coupler for nondiffracting CGB, we can consider the interferogram of a normally incident plane wave and two SPPs plane waves propagating at a half angle $\theta_{\mathrm{CGB}}$, figure $7(a)$. We observe that the zero-intensity lines in figure $7(b)$ follow those of the two intersecting gratings $(c)$ used to excite non-diffracting cosine Gauss plasmon beams. An example of the near-field distribution of the CGB, measured via near-field optical microscopy (Lalouat et al 2011), is presented in figure $7(d)$. Due to the finite size of the holographic coupler, the two plasmon beams interact over a finite spatial region. Cosine-Gauss non-diffracting transverse intensity distribution presented in (e) would exist therefore only throughout the overlap region. The long range propagation of the CGB reported in Lin et al (2012) is only due to the geometry of the excitation grooves. The latter are disposed at an angle so that fields interfering along the symmetry axis propagate a distance which is in fact shorter than the CGB 'effective propagation' distance.

A design modification that gives increased transverse localization, i.e. with less intensity in the side lobes is shown in figure 8 and was found to exhibit non-diffracting properties as well, see Lin et al (2012). The advantage of this SPP beam (which is called the localized cosine-Gauss Beam (LCGB)) for applications is the increased transverse confinement compared to the CGB, while maintaining negligible diffraction. One can in fact show that the device of figure 8 can generate LCGBs with axial intensities much larger than conventional SPP beams launched by a single grating of comparable transverse dimension. In this specific case, the plane wave couplers were arranged such that they don't spatially overlap, but one can use the holographic approach to calculate a more compact structure. By analogy to the $3 \mathrm{D}$ optical bottle beam (Arlt and Padgett 2000, McGloin et al 2003, Yelin et al 2004), a plasmonic bottle beam which features a lattice of plasmonic bottles, i.e. alternating regions of bright focii surrounded by low intensities, has recently been introduced (Genevet et al 2013).

Compact couplers for non-diffracting Airy beams (Siviloglou and Christodoulides 2007, Siviloglou et al 2007) have also been introduced following the holographic approach (Dolev et al 2012), demonstrating for the first time the conversion of SPPs into an Airy free space beam. The Fourier transform of the Airy function which is cubic as a function of the spatial coordinates defines where we have to etch lines of apertures to scatter SPP into the desired farfield. It is possible to follow this design technique to create surface waves with arbitrary wavefronts such as non-diffracting Airy plasmon beams (Salandrino and Christodoulides 2010, Minovich et al 2011, Zhang et al 2011) or even more complex near-fields (Klein et al 2012, Itai Epstein 2014). The properties of these plasmonic fields make them suitable for experimental implementation of plasmonic trapping (Righini et al 2007, Juan et al 2011, Wang et al 2011, Wang and Crozier 2012). 

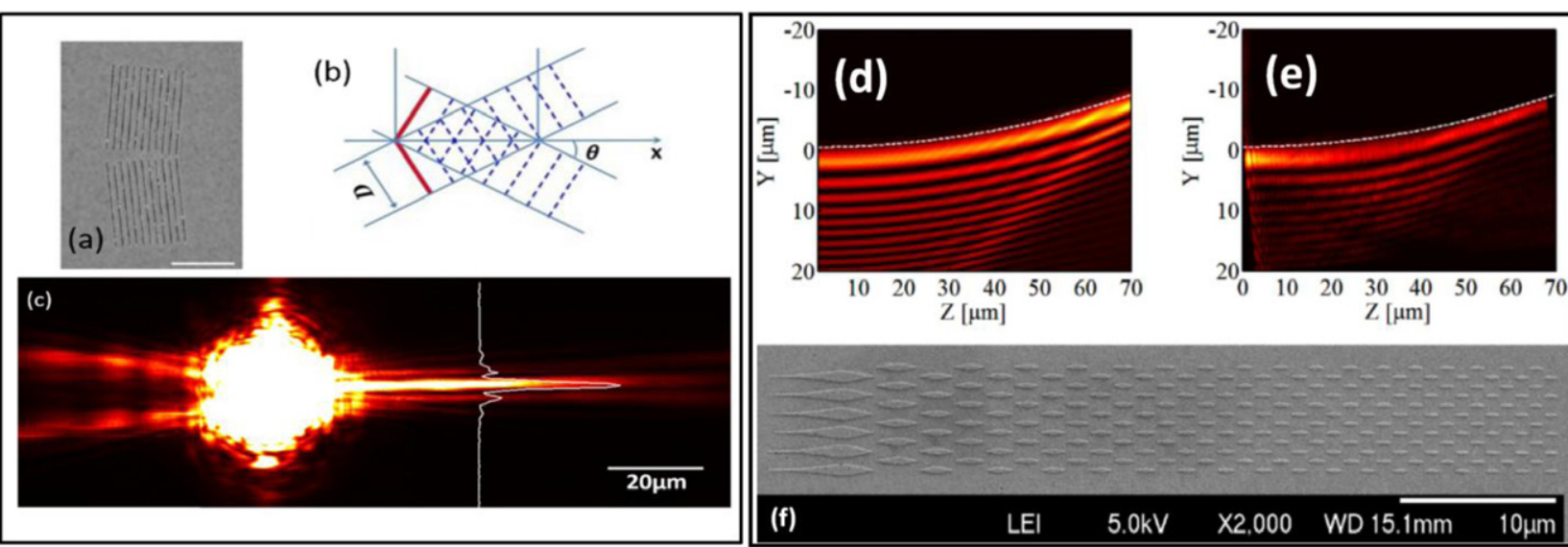

Figure 8. The localized cosine-Gauss beam (LCGB) generated by two gratings at an angle as shown in $(a)$ and $(b)$. The white bar is (a) represents 5 microns. The near-field intensity distributions (in-plane components) experimentally with an NSOM; overlaid in white, transverse intensity distributions at specific propagation distance. A part of the figure is reproduced with permission from Lin $e t a l$ (2012). $(d)$ simulation and $(e)$ NSOM measurement of plasmonic Airy beam. $(f)$ SEM image of the plasmonic hologram the generated the plasmonic Airy beam. Figure reproduced with permission from Itai Epstein (2014).
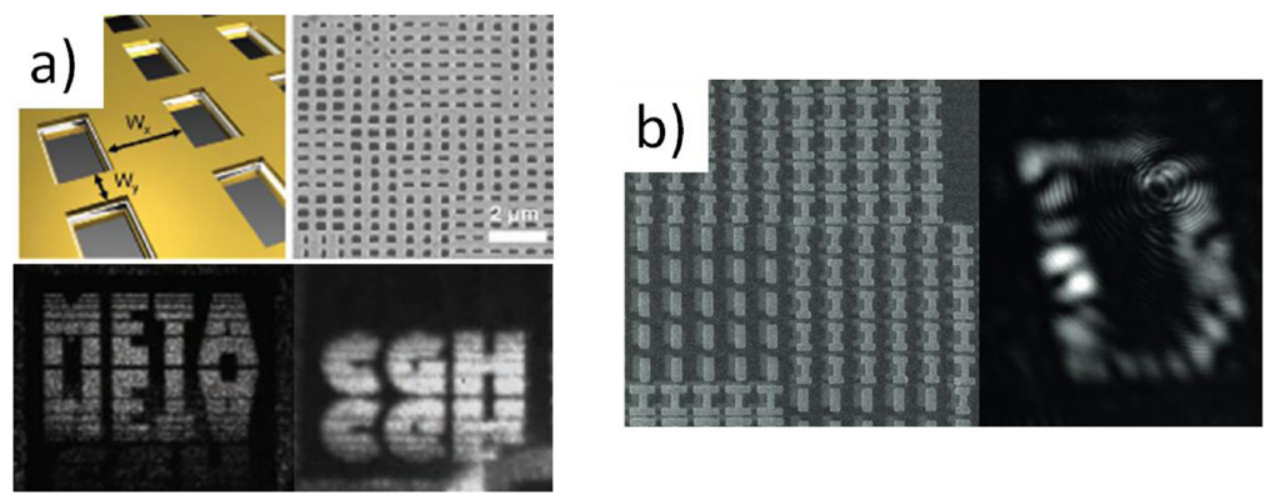

Figure 9. Examples of layered metamaterial holograms. (a) Engineering the dispersive properties of few layers of a fishnet metamaterials, it is possible to produce two holographic images at two different wavelengths. (b) Computer-generated phase hologram created by assembling multilayer of lithographically patterned I-beam metallic inclusions. These results have been obtained in the near and midinfrared frequency range. The figures are reproduced with permission from (a) Walther et al (2012) and (b) Larouche et al (2012).

\section{Binary nano-structured detour phase holograms}

The ultimate challenge for manipulating a light beam is the control with a single optical element of the spatial distribution of amplitude, phase and state of polarization. Few techniques, such as detour-phase holograms, achieve both amplitude and phase modulation with a single component. Thin metamaterials holograms have been proposed as the first step towards the development of a new class of versatile sub-wavelength thick optical components capable of controlling amplitude, phase and polarization at a given frequency. The latter are created by assembling layers of interfaces, each covered or etched with nanostructures (figure 9). The interested reader may refer to the following: Larouche et al (2012), Walther et al (2012).

Very recently, we witnessed the development of a new type of optical interfaces, i.e. a single layer of resonant elements or metasurfaces, that can introduce abrupt phase jumps along the propagation of light to manipulate phase, amplitude and eventually the polarization of light over subwavelength propagation distances (Yu et al 2011, Genevet et al 2012, Ni et al 2012, Yu et al 2012, 2013, Pors et al 2013, Genevet 2014, Yu and Capasso 2014). This can be performed by controlling precisely the position and the scattering properties of nanoscale optical resonators patterned at the interface. The metasurface approach provides great flexibility in the design of the wavefront of light and can be applied to create surface holograms (Huang et al 2013, Ni et al 2013, Zhou et al 2013, Chen et al 2014 and references therein). Examples of these surface holograms, made of metallic nanostructures that convert incident free space beams into free space objects, are presented in figure 10. The scattering properties of the different nanostructures are in general wavelength and polarization sensitive, thus by leveraging on the polarization response of nano-structured elements at interfaces, one can design polarization and wavelength sensitive holographic interfaces, see for example Wei Ting Chen et al (2014), Montelongo et al (2014) and Huang et al (2013). Moreover, the size of resonant nanoparticles can be much smaller than the wavelength and one can therefore design multiwavelength and polarization sensitive surface holograms. These new types of devices would open up 


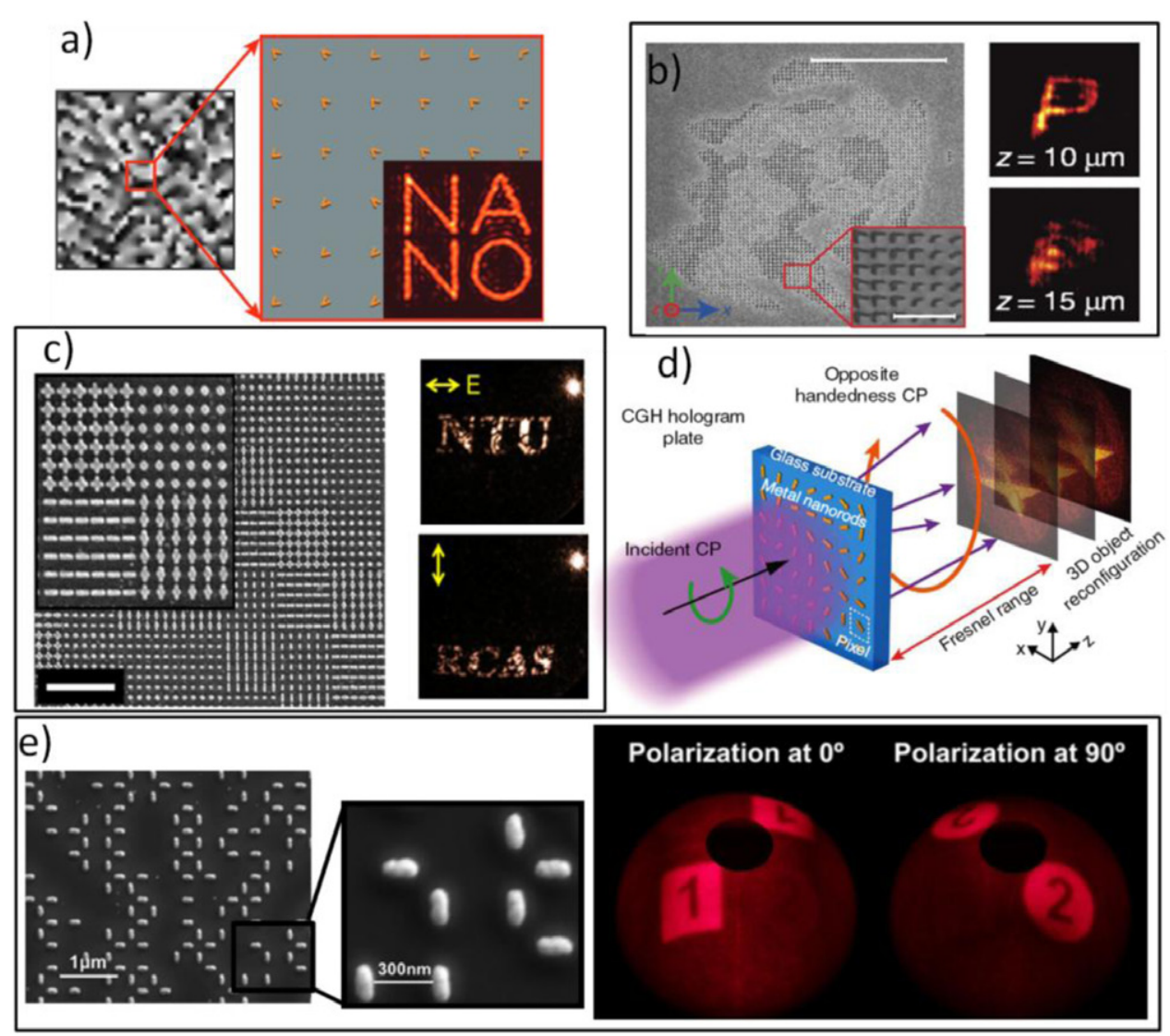

Figure 10. (a) Numerical demonstration of planar hologram based on the concept of resonant nanoantenna metasurfaces. (b) Experimental demonstration of an holographic metasurface. $(c)-(e)$ are examples from the literature on polarization controlled-holograms. The figures are reproduced with permission from Huang et al (2013), Ni et al (2013), Zhou et al (2013), Montelongo et al (2014), Chen et al (2014). Note that in the work in $(e)$, the wavelength approaches the period and higher-order Floquet-Bloch modes have to be considered, drawing the line between metasurface holograms and purely diffractive holograms.

new types of applications in photonics. So far, metasurfaces have poor efficiencies which hinder using this approach for real applications. New techniques that could overcome these limitations, for example by cancelling reflection ${ }^{7}$ and by reducing optical losses ${ }^{8}$, have still to be investigated and applied to create surface holograms.

Allying some concept of nanophotonics with the principle of detour-phase holography, we have proposed a different type of holographic plates which after being designed initially for a given wavelength, can manipulate phase amplitude and polarization over a significant part of the visible spectrum (Lin et al 2013). The novelty lies in replacing the large openings of conventional holograms by arrays of subwavelength apertures, oriented to locally select a particular state of polarization. The resulting design can therefore be viewed as the superposition of two independent structures with very different length scales, i.e. a detour-phase hologram with each of its apertures carved with nanoscale openings to transmit only

\footnotetext{
${ }^{7}$ See the recently proposed Huygens' interfaces Pfeiffer and Grbic 2013 Metamaterial Huygens' surfaces: tailoring wave fronts with reflectionless sheets Phys. Rev. Lett. 110 19. Monticone et al Ibid Full control of nanoscale optical transmission with a composite metascreen (20).

${ }^{8}$ Using for example dielectric resonator metasurfaces Decker M, Staude I, Falkner M, Dominguez J, Neshev D N, Brener I, Pertsch T and Kivshar Y S 2014 High-efficiency light-wave control with all dielectric optical Huygens metasurfaces (arXiv:1405.5038).
}

a desired state of polarization. Before discussing these new nano-structured holographic plates, we would like to give a brief overview on the design of conventional detour phase holograms.

\subsection{Detour phase holograms}

The concept of traditional detour-phase hologram, introduced by Brown and Lohmann in 1966 (Brown and Lohmann 1966), can be illustrated by considering an infinite $1 \mathrm{D}$ structure made of periodically repeated apertures of width $\alpha W$ with a period $W$, where $\alpha \leq 1$. The transmittance function in the plane in $z=$ 0 of this structure is given by:

$$
T(x)=\left(\sum_{n} \delta(x-n W)\right) * \prod\left(\frac{x}{\alpha W}\right)
$$

where the asterisk denotes convolution and $\Pi$ denotes the 'top hat' function. The diffraction pattern of this function is given, in the paraxial approximation, by:

$$
f(\theta)=\frac{\mathrm{e}^{-j k z}}{j \lambda z} \widehat{T}\left(\frac{\sin (\theta)}{\lambda}\right)
$$

where the symbol ${ }^{\wedge}$ denotes the Fourier transform and $z$ is the direction normal to the hologram. By shifting the periodic 
(a)

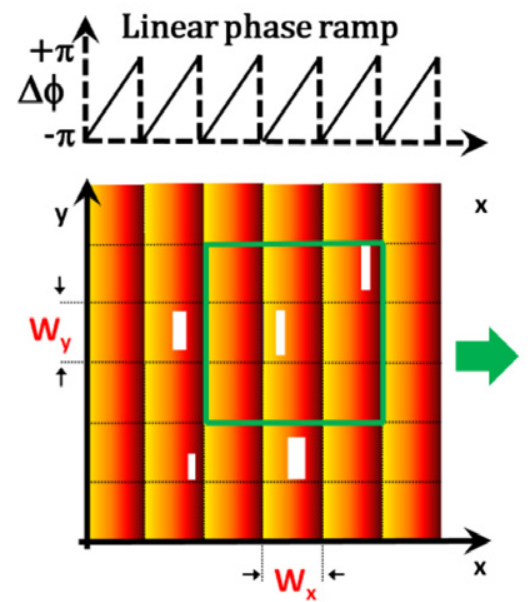

(b)

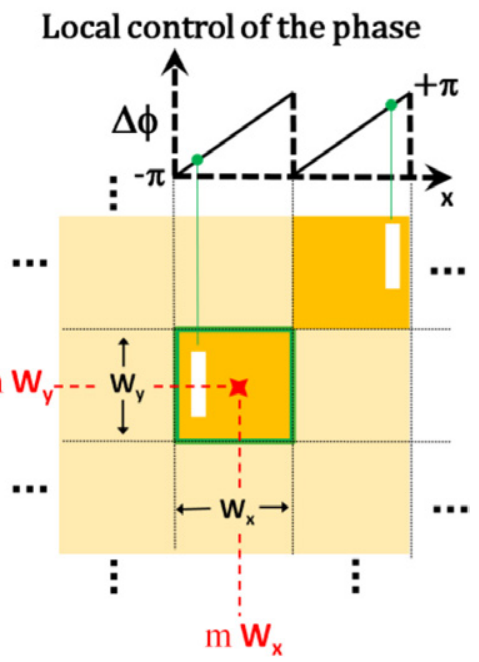

Figure 11. Schematics $(a)$ and $(b)$, without any subwavelength apertures, illustrate the concept of conventional detour-phase holograms. (a) Consider a monochromatic plane wave incoming at normal incidence and transmitted through the apertured interface: from the shift theorem of Fourier optics, an aperture shifted by $\delta_{n m}$ from the center of the $n m$-th pixel creates a phase shift given by $2 \pi \delta_{n m} x / \lambda z=2 \pi \delta_{n m} / \lambda \sin (\theta)$ where $z$ is the direction normal to the hologram, i.e. an interface seen by an observer standing at an angle $\theta$ from the normal can be discretized by sections of width $W_{x}=\lambda / \sin (\theta)$, each of which can then be apertured to select a phase within the $2 \pi$ range. Conventional detour-phase holograms are composed of many pixels, each scattering light with a given phase delay. $(b)$ The overall complex amplitude of light emerging through each pixel is designed by controlling both the position and the size of the slit in the pixel. The width of the apertures, $\alpha$, is kept constant across the interface. The amount of light passing through the pixel at $\left(m W_{x}, n W_{y}\right)$ is then proportional to the length of the aperture $\beta_{m, n}$. The aperture $x$-position within the pixel $\left(\delta_{m, n}\right)$ controls the phase of the light transmitted through the $(n, m)$ th pixel.

function along the lateral dimension $x$ by an amount of $\delta$ a, i.e. $T=T(x-\delta)$, the shift theorem of Fourier optics implies that the nonzero diffraction orders experience a phase oscillation equal to $\mathrm{e}^{2 j \pi \delta \sin (\theta) / \lambda}$ (Goodman 1996). This factor is easily understood physically by noting that waves diffracted by adjacent slits give rise to intensity maxima in a direction $\theta$ if their path difference along $\theta$ is an integer number of wavelengths. The detour-phase holograms, which are basically apertures etched in opaque films, use this property to encode phase and amplitude of the wavefront. The apertures are disposed along the $x$ direction in the $z=0$ plane, at locations $\delta$ to select the desired phase shifts, see figure 11(a). Note that at an angle $\theta_{1}$, the phase shift for a given wavelength $\left(\lambda_{1}\right)$, will be observed at another wavelength $\left(\lambda_{2}\right)$ at the angle $\theta_{2}$ defined by $\theta_{2}=\sin ^{-1}\left[\lambda_{2} / \lambda_{1} \sin \theta_{1}\right]$.

The overall design of a detour phase hologram can therefore be understood by recognizing that the apertures are equivalent to the lines of maximum (or minimum) of intensity of an interferogram produced by interfering a reference plane wave incident at an angle $\theta$ denoted by $E_{\text {ref }}(x, y)=A \mathrm{e}^{-\mathrm{i} k \cdot r}$, in which $k_{x}=2 \pi / W_{x}$, with an object wave $E_{\text {obj }}(x, y)$ (Lee 1979). We refer the reader to Tricoles (1987) for an interesting historical review on the development of this technique.

\subsection{Holograms with sub-wavelength apertures}

To this method of manipulating phase and amplitude (which is controlled by the size of the apertures) we can add control over the spatial distribution of the polarization by including subwavelength structures within the apertures of the detourphase hologram, as illustrated in figure 12.
To avoid spurious diffraction effects from these polarizing metallic apertures, their size is chosen to be significantly smaller than the wavelength of light. The resulting design can therefore be viewed as a superposition of two independent structures with very different length scales. The larger scale features are spaced by roughly $\lambda / \sin \theta$, where $\theta$ is the first order diffraction angle for normal incidence; and as we discussed they control the spatial modulation of the phase. The smaller features can be viewed as miniature wire-grid polarizers, which locally control the state of polarization of the transmitted light. Given an incident beam with a generic state of polarization (i.e. elliptical) incident on a 2D apertured interface, the transmitted field distribution is $\vec{E}_{z=0^{+}}(x, y)=\vec{E}_{z=0^{-}}(x, y) T(x, y)$ where $z=0^{+}$and $z=0^{-}$represent the location just ahead and just behind an interface represented by a transmittance function $T(x, y)$. Assuming an incident circularly polarized beam:

$$
\overrightarrow{E_{\mathrm{cp}}}=\overrightarrow{e_{x}}+j \overrightarrow{e_{y}}=\mathrm{e}^{j \theta}\left(\overrightarrow{e_{r}}+j \overrightarrow{e_{\theta}}\right)
$$

the transmittance $T(x, y)$ in a vectorial form reads:

$$
\begin{gathered}
T(x, y)=\left(\sum_{n, m} \delta\left(x-n W_{x}-\delta_{n m}, y-m W_{y}\right)\right) * \\
\prod\left(\frac{x}{\alpha W_{x}}, \frac{y}{\beta_{n m} W_{y}}\right)\left[\begin{array}{c}
\cos \varphi_{m n} \\
\sin \varphi_{m n}
\end{array}\right]
\end{gathered}
$$

The parameters $\alpha$ and $\beta_{m n}$ which represent the size of the opening along $x$ and $y$ within a single cell, control the amplitude and the phase of the scattered field. In particular, the local phase shift at the pixel $(m, n)$ is given by $\varphi_{m, n}=2 \pi \delta m n / W_{x}$ and the 


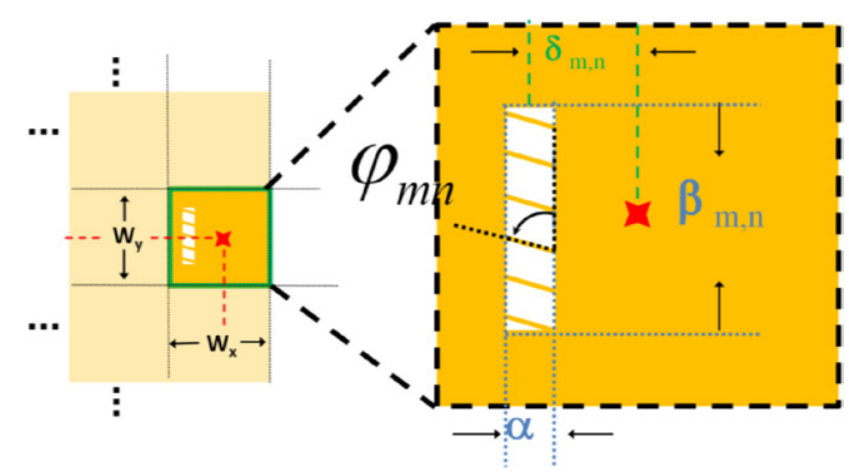

Figure 12. By decorating each aperture in figure $14(b)$ with subwavelength features to form a wire-grid polarizer oriented at some angle $\varphi_{n, m}$, we can locally convert a generic state of polarization (i.e. elliptical) of incident polarization into a specific state of polarization of the scattered radiation For linear apertures with large aspect ratio, the selected polarization is linear.

amplitude increases monotonically with increasing $\beta_{m n}$. It is important to point out that the width of the slit in the x-direction, noted $\alpha$ in figure 12 not only affects the transmitted amplitude but also affects the precision with which the phase is specified. Precise phase selection ideally requires very narrow slits, which, in practice, considerably decreases the amount of light transmitted through each pixel. In our experiments, a compromise is reached for $\alpha / \mathrm{W}_{\mathrm{x}}=0.5$, i.e. when half of the area of the element is transparent, as previously demonstrated for binary holograms (Lohmann and Paris 1967).

\subsection{Pancharatnam-Berry phase}

Spatial control of the polarization state of a light inevitably introduces unwanted spatially-varying phase distributions, known to be a manifestation of the Pancharatnam-Berry (P-B) phase (Pancharatnam 1956, Berry 1984, Zhan and Leger 2002). Unlike diffractive and refractive elements, geometric phase retardations can be created by introducing space-variant polarization manipulation of the incident field. This was performed by modifying the orientation of sub-wavelength micro/ nanostructured dielectrics in a space-variant fashion (Bomzon et al 2002), leading to the design of new optical phase elements in which the phase is not addressed via optical path differences (or via the resonant properties of nano-structured optical resonators, as discussed above). Computer-generated holograms employing spatial phase retardation of subwavelength gratings with desired orientation and birefringence has also been experimentally demonstrated (Levy et al 2004, 2005), see figure 13.

Phase modulation and polarization modulation are not independent features that can be simply added to each other. Therefore, the approach for designing holographic interfaces to project any vectorial holographic images is more than just a simple superposition of wire grid polarizers to conventional detour-phase holograms. As we will show in the following for the generation of radially polarized beams, the $\mathrm{P}-\mathrm{B}$ phase induced by the projection of the incident polarization onto the desired state of polarization plays an important role and has to be determined in advance, before designing the holograms.

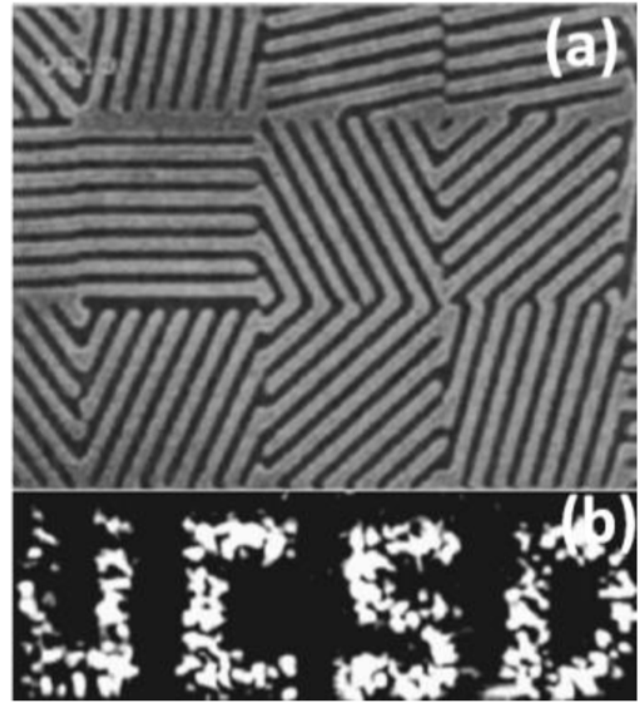

Figure 13. (a) Typical SEM photograph of the fabricated birefringent computer-generated holograms $(b)$ Experimental Far-field reconstruction of the hologram for $1.55 \mu \mathrm{m}$. Figures reproduced with permission from Levy et al (2005).

The detour-phase which is defined by the position of the aperture in each pixel has to be equal to the difference between the desired phase modulation and the $\mathrm{P}-\mathrm{B}$ phase.

\subsection{Application to the generation of radially polarized beam}

In the following, we shall discuss a particular implementation of these nano-structured holograms to create a radially polarized beam from an incident circularly polarized Gaussian beam. Significant efforts have been devoted to the creation of cylindrical vector beams (CVBs), a family of solutions of the more general vector wave equation (Hall 1996), featuring a cylindrical state of polarization in the dimension transverse to the propagation direction(Zhan 2009). CVBs are familiar subjects in electromagnetic waveguide theory and are often encountered in metallic waveguides with cylindrical boundary conditions or in optical fibers. One can also directly produce free-space CVBs from lasers by imposing an appropriate transverse mode selection mechanism (Pohl 1972).

The radially polarized beam (RPB) is the most widely used free-space CVB (figure 14) (Tidwell et al 1990, Volpe and Petrov 2004, Niziev et al 2006, Novotny and Hecht 2006, Ahmed et al 2007, Grosjean et al 2008, Lou et al 2012). Due to complete radial symmetry in their transverse field, including the polarization state, RPBs have potential for applications in imaging (Dorn et al 2003, Sheppard and Choudhury 2004, Wang et al 2008), optical trapping (Zhan 2004, Zhao et al 2005, Kawauchi et al 2007) and laser machining (Niziev and Nesterov 1999, Kraus et al 2010). A radial polarizer, which consists of subwavelength circular metallic gratings as shown in figure 15(a) (Ahn et al 2005), has recently been used to generate an RPB from a circularly polarized input beam (Ghadyani et al 2011). From equation (13), one recognizes that attempting to convert a circularly polarized beam into a RPB by using a radial polarizer yields a far-field intensity pattern that deviates 

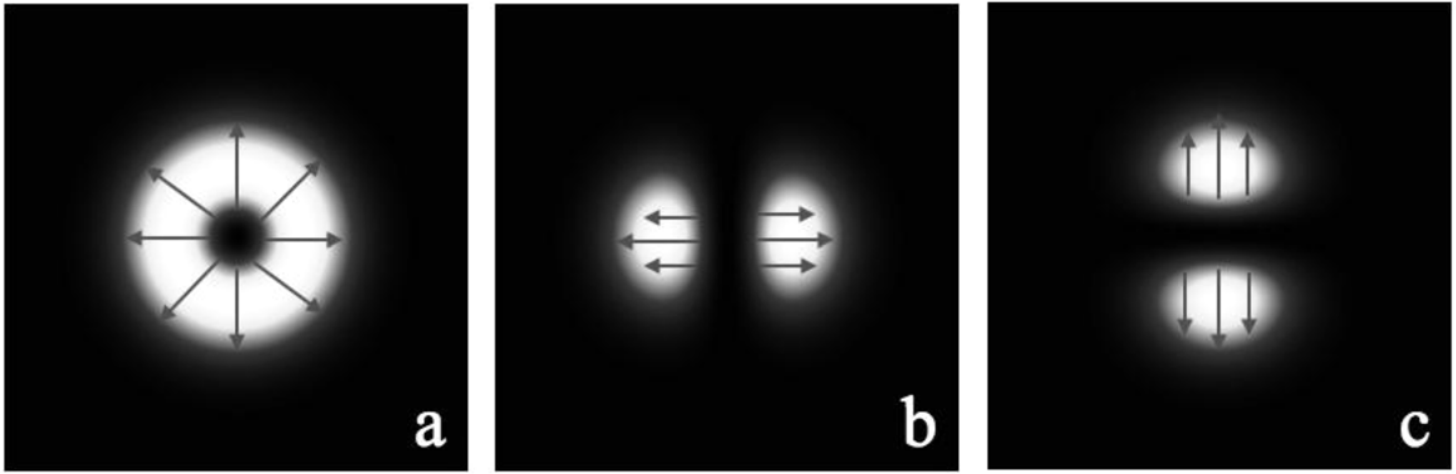

Figure 14. (a) The hollow intensity distribution of a typical radially polarized beam (RPB). The RPB mode can be decomposed in a linear combination of two orthogonal $x$ - and $y$-polarized $\mathrm{HG}_{10}$ modes. The intensity distribution of the $\mathrm{HG}_{10}$ modes are shown in $(b)$ and $(c)$. The blue arrows indicate the state of polarization. Figures reproduced with permission from Lin et al (2013).
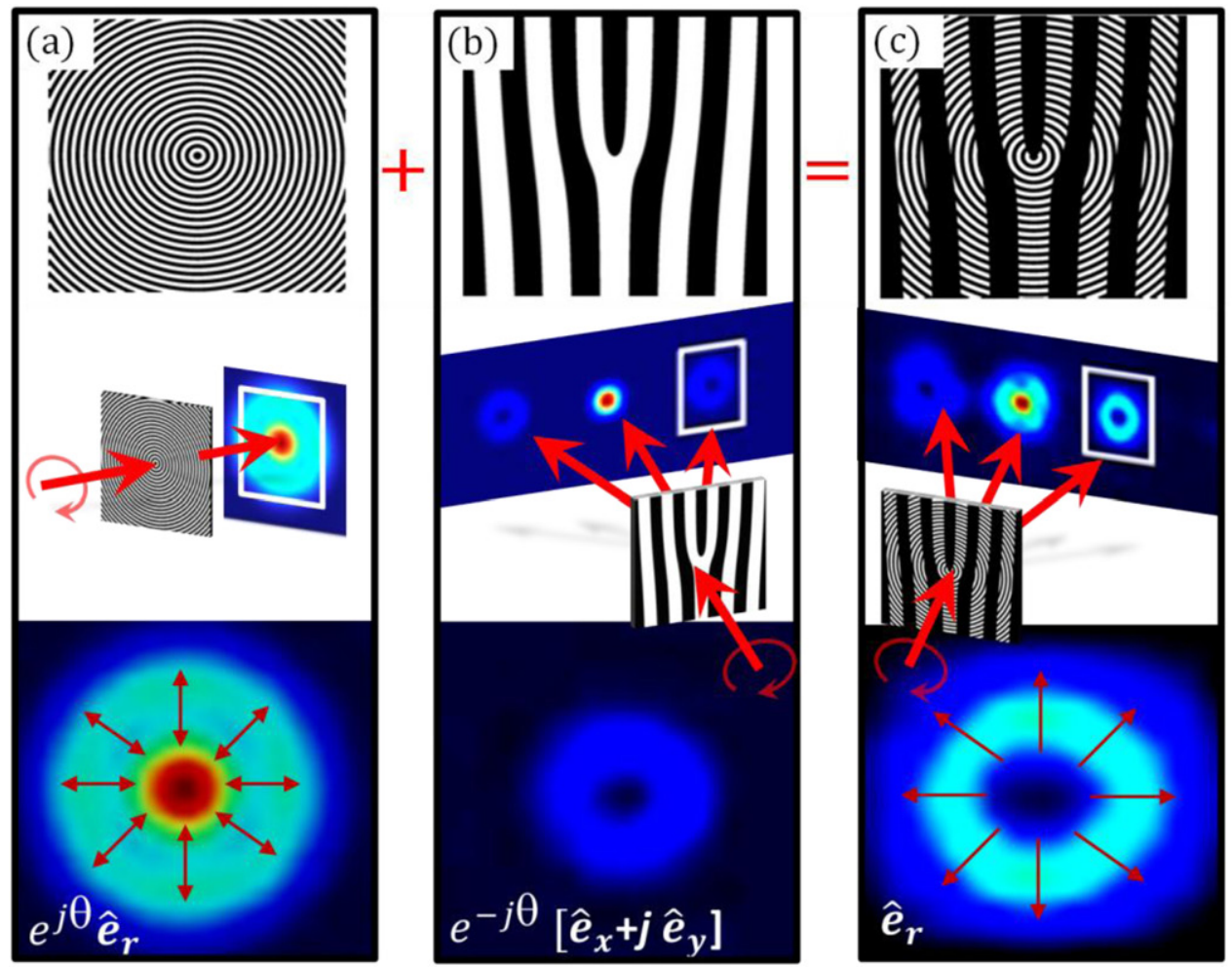

Figure 15. The technique used to generate radially polarized beams (RPBs) combines sub-wavelength apertures for polarization control and wavelength-scale diffracting apertures. The structure that generates RPBs $(c)$ is the superposition of a radial polarizer $(a)$ and a fork diffraction hologram $(b)$ to cancel the $\mathrm{e}^{j \theta}$ phase contribution that arises from the projection of the circularly polarized optical field onto the state of radial polarization, as explained in the text. The black and white colors in the upper row represent respectively ' 0 ' (opaque) and ' 1 ' (transparent) in the transmittance function of the device. The periods in $(a)$ and $(b)$ are $200 \mathrm{~nm}$ and $1.2 \mu \mathrm{m}$, respectively. For each panel, the figure in the middle presents the far field intensity distribution after traversing the structure with the transmittance function given by the upper part of the panel. The simulated interfaces comprise patterned Au films (150 nm thick) on a glass substrate. The calculated far-field intensity distributions presented in the lower part of each panel are enlarged versions of the diffracted beams located in the white boxes. Each sample is illuminated by a right-handed circularly polarized plane wave incoming at normal incidence. The wavelength of the light in the simulation is $633 \mathrm{~nm}$, but identical results have been obtained across the visible spectrum. All simulations have been performed using commercial finite-difference time-domain (FDTD) software. Figures reproduced with permission from Lin et al (2013).

from the desired doughnut shaped RPB. An example of the farfield intensity distribution is presented in figure $15(a)$.

The additional phase imparted to a light beam in the process of polarization conversion from a circularly-polarized beam to a radially-polarized one using a radial polarizer has a spiral distribution $\mathrm{e}^{j \theta}$. The origin of this spiral phase is the projection of the incident light with, for example, right-handed circular polarization (equation (13)) onto the state of polarization of the light passing through a circular wire-grid polarizer, i.e. the radial state of polarization, such that the output beam profile can be described by:

$$
\overrightarrow{E_{\text {out }}}=\mathrm{e}^{j \theta} \overrightarrow{e_{r}}
$$

The P-B phase term, $\mathrm{e}^{\mathrm{j} \theta}$ in equation (15), is often compensated by additional optical elements, e.g. spiral phase plates (Beijersbergen et al 1994) that impose a conjugate spiral 

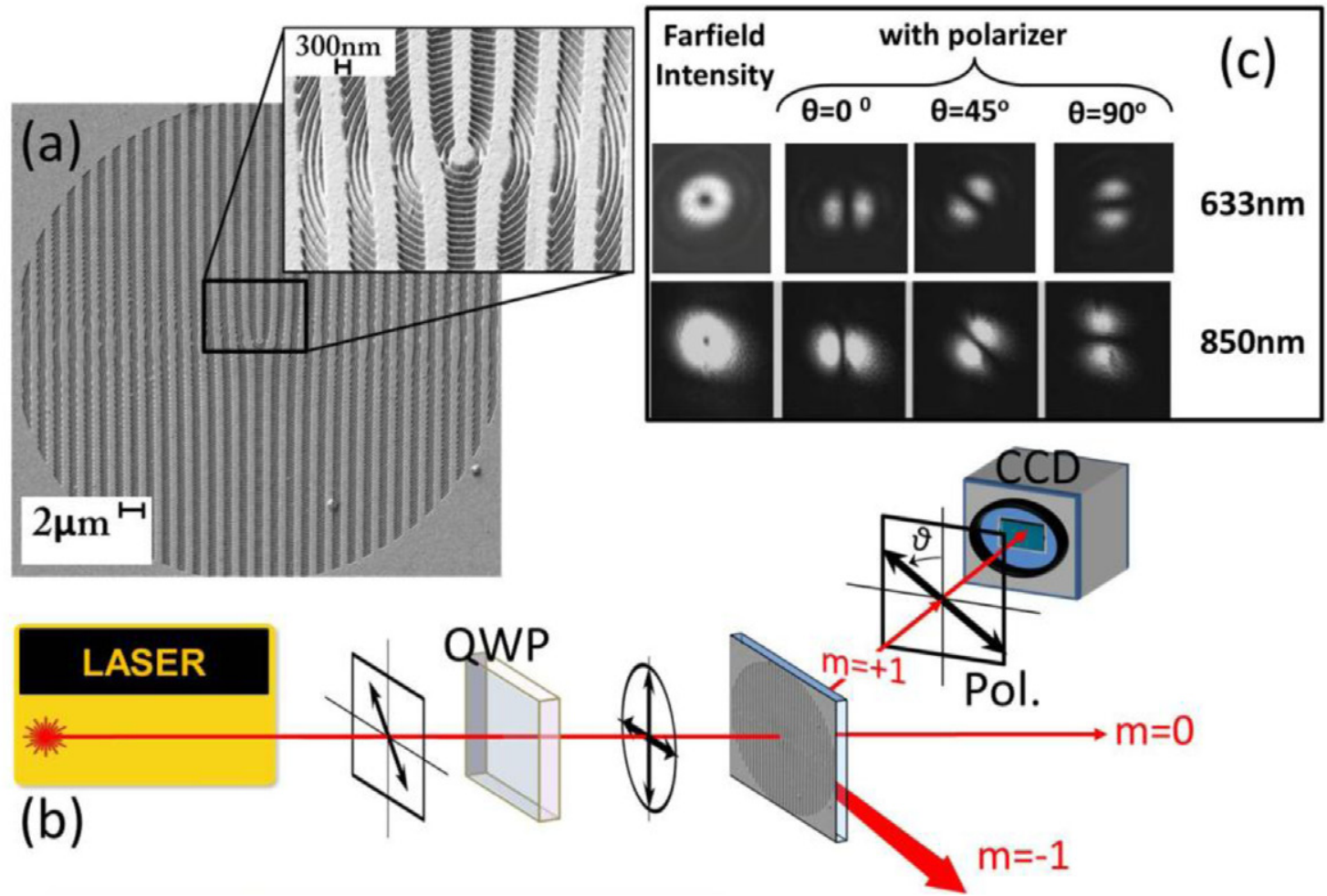

Figure 16. The fabricated structure $(a)$ was tested with two different wavelengths $(633 \mathrm{~nm}$ and $850 \mathrm{~nm})$. A quarter wave plate converts the linearly polarized laser beam to a circularly polarized one. The far-field distribution is captured by a CCD camera after passing through a linear analyzer oriented at varying angles. (a) SEM micrograph of the device; $(b)$ the experimental set-up; $(c)$ measured far-field intensity distributions at two wavelengths, without and with analyzer, the latter oriented at different angles in front of the CCD camera. These intensity patterns demonstrate that the $m=+1$ beam is radially polarized and that device is broadband. Note that the sample creates several diffraction orders; due to the subwavelength patterning, the $m=-1$ order acquires an additional orbital angular momentum, leading to an overall $\mathrm{e}^{\mathrm{j} 2 \theta}$ phase factor. Figures reproduced with permission from Lin et al (2013).

phase $\mathrm{e}^{-j \theta}$, i.e. with the helicity of wavefront reversed with respect to the unwanted $\mathrm{P}-\mathrm{B}$ phase. A different approach to introduce such a spiral phase distribution involves diffracting the light from a binary fork hologram (figure 15(b)), which achieves phase modulation in the diffracted orders (Bazhenov et al 1990, Heckenberg et al 1992). We discussed previously that the design of such a fork hologram can be performed by calculating the off-axis interference pattern between an object vortex beam and a reference beam (usually a plane wave or Gaussian beam). To generate a RPB, the fork hologram creates a spiral phase front, and the subwavelength apertures select the radial state of polarization. For incident light with circular polarization, the overall effect of the structure is to generate a radially polarized beam without the need for an additional $\mathrm{P}-\mathrm{B}$ phase compensation element, figure $15(c)$.

To demonstrate our technique, we fabricated a binary flat optical component to generate an RPB from a circularly polarized Gaussian beam by ion milling the pattern shown in figure 16(a), using a focused ion beam (Zeiss NVision 40), into a $150 \mathrm{~nm}$-thick Au film deposited by electron beam evaporation onto a glass substrate. The period of the subwavelength apertures is $200 \mathrm{~nm}$ and the size of the features forming the hologram is approximately $1.25 \mu \mathrm{m}$. As shown in figure $16(b)$, our sample was illuminated by circularly polarized laser beams at two different wavelengths $(633 \mathrm{~nm}$ and $850 \mathrm{~nm})$. The diffraction order that carries the RPB $(m=+1)$ was directed through a linear polarizer and its intensity distribution was captured by a CCD camera. By rotating the linear polarizer in front of the beam with the characteristic doughnut intensity profile $(m=$ +1 diffraction order), we demonstrate in $(c)$ that its polarization is oriented radially, in agreement with figure 14. Although these holograms are designed to create a virtual image at a given angle for a specific wavelength, they actually operate over a broad wavelength range, creating virtual images at the corresponding angles, see figure 16(c).

The devices proposed in this letter are still related to traditional detour-phase holograms, and suffer from the same low output efficiency: only one (defined as $m=+1$ ) of the two first diffraction orders carries the desired amplitude, phase and polarization which gives rise to the RPB. Similar to Leith-Upatnieks holograms (Leith and Upatniek 1962), a twin image with conjugate phase modulation exists in the other first order. This second beam carries a phase factor $\mathrm{e}^{2 j \theta}$ corresponding to an additional angular momentum acquired in scattering off the holographic grating.

\subsection{Polarization sensitive directional holograms}

Recently, polarization dependent directional launching of SPP has been reported in nanostructured 1D gratings. The directional coupling mechanism opens up new opportunities for developing polarization sensitive directional 

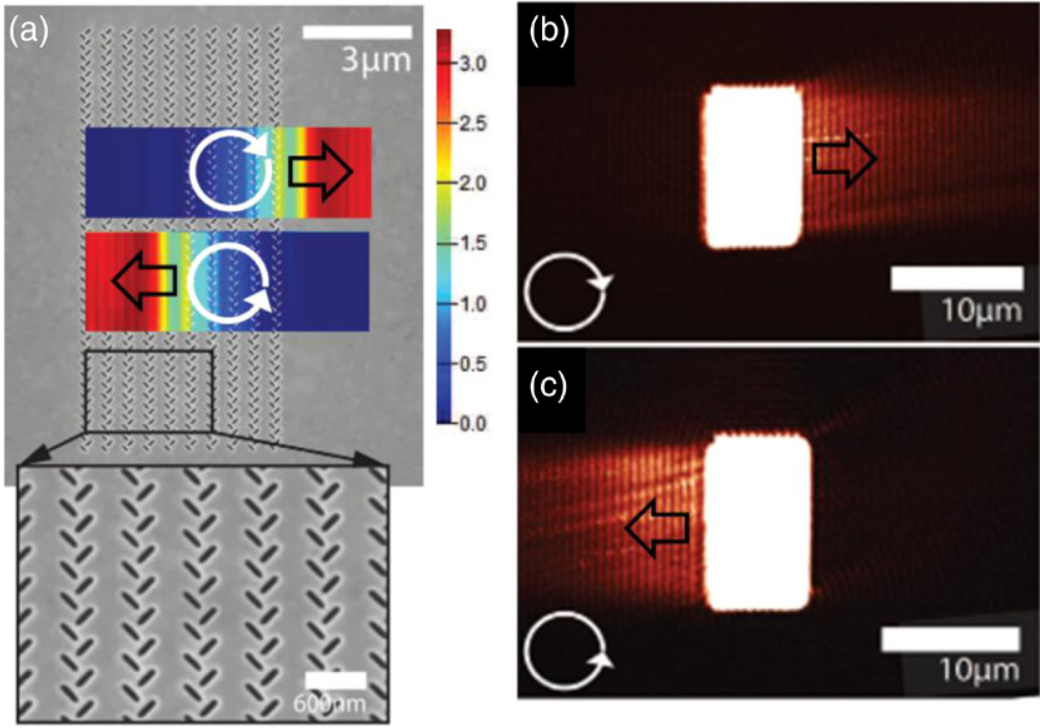

Figure 17. Measurement of the SPPs launched by a fabricated coupler. (a) Scanning electron microscope (SEM) image of a structure fabricated in a gold film for operation at $\lambda=633 \mathrm{~nm}$. Overlaid on the SEM image, we present the FDTD simulation of the near-field intensity (arbitrary units) above a coupler made up of five column pairs under illumination by incident light with circular polarizations (white arrows). (b) and (c) NSOM images of the structure under illumination from the back by right CPL (b), and left CPL (c). The state of incident polarization is shown by white arrows. The fringes arise from interference between the transmitted incident beam through the gold film and the propagating SPPs. Figures reproduced and adapted with permission from Lin et al (2013).

holograms. When an array of apertures in metallic films is arranged in a column with a spacing that is smaller than the SPP wavelength, the launched SPPs are plane waves that propagate perpendicularly away toward either side of the column and emission is obtained independently from the orientation of the dipoles of the column. Therefore it is possible to create a source of SPP plane waves propagating in a fixed direction that can be designed to selectively respond to a polarization component of the incident light, the latter is determined by the orientation of the apertures of the column. Polarization sensitive holographic plasmon couplers can be designed by considering the interference of the SPP waves created by several columns, each having a specific polarization response and arranged following a holographic design discussed in the second paragraph of this paper. For example, it is possible to create a directional 1D plasmonic coupler by considering a periodic array of two parallel columns, spaced a sub SPP wavelength distance apart and made up of apertures with different orientation. For this specific coupler presented in figure 17 and discussed in detail in Lin et al (2013), the spacing between the two sub units is equal to a quarter of the SPP wavelength and the apertures of the first and second column are oriented at $90^{\circ}$ with respect to each other. If light is normally incident on the metal surface, the respective columns will then couple to the two normal components of the incident field. Using circular polarization, the phase retardation between the two normal components transferred to the SPP differs by a phase factor $\mathrm{e}^{\mathrm{i} \sigma \pm \pi / 2}$, where the spin angular momentum $\sigma \pm= \pm 1$ whether light is left of right circularly polarized. The propagating phase accumulated by SPP propagating over a distance of a quarter of SPP wavelength is $\mathrm{e}^{-\mathrm{i} \sigma \pm \pi / 2}$ depending the direction of propagation. Therefore, constructive interference of the SPP waves launched by each column results in time-averaged SPP field intensities propagating to the right or to the left to the pair of columns, depending on the sign of the spin-angular momentum of light.

\section{Conclusions}

In summary, we have discussed a holographic approach to model and to design any sort of 2D metallic grating. Using this model, it is possible to design a new class of plasmonic devices, capable of producing a large variety of free-space beams and also capable of launching surface plasmon polaritons from incident free space beams with unusual amplitude and phase distributions. As examples of applications, we demonstrated dual-beam emission from mid-infrared quantum cascade lasers, the sorting of the orbital angular momentum of light and the generation of plasmonic nondiffracting cosine and Airy beams. The holographic method of generating plasmonic couplers has potential for applications in various areas of integrated optics and can be used, in a straightforward way, to design any other sort of interface.

In the last part of this review, we show that by carving the apertures of detour-phase holograms with subwavelength features we can manipulate complex scalar fields. In particular, by incorporating subwavelength wire-grid polarizer, we achieved full control of vector optical field over a broad range of wavelengths with a flat sub-wavelength binary structure. The ability to appropriately shape amplitude, phase and polarization of light with single ultrathin interfaces gives an incentive for the generation of more complex light fields such as high-order Bessel, Mathieu or Airy beams and promises 
interesting advances in the fields of integrated photonics, optical trapping or quantum optics.

\section{Acknowledgments}

The authors thank L Jiao, R Blanchard, J-P Tetienne, B Cluzel, J Dellinger, M A Kats, T Mansuripur and F De Fornel for discussions and materials. This research is supported in part by the EArly-concept Grants for Exploratory Research (EAGER) at NSF Grant (ECCS-1347251). The authors also acknowledge support from the Harvard Nanoscale Science and Engineering Center (NSEC) under contract NSF/PHY 06-46094. This work was supported in part by the Defense Advanced Research Projects Agency (DARPA) N/MEMS S\&T Fundamentals program under Grant N66001-10-1-4008 issued by the Space and Naval Warfare Systems Center Pacific (SPAWAR) and by the Air Force Office of Scientific Research under grant number FA9550-12-1-0289. Fabrication of the devices was carried out at the Center for Nanoscale Systems (CNS), a member of the National Nanotechnology Infrastructure Network (NNIN).

\section{References}

Ahmed M A et al 2007 Multilayer polarizing grating mirror used for the generation of radial polarization in Yb: YAG thin-disk lasers Opt. Lett. 32 3272-4

Ahn S W et al 2005 Fabrication of a $50 \mathrm{~nm}$ half-pitch wire grid polarizer using nanoimprint lithography Nanotechnology 16 1874-7

Arlt J and Padgett M J 2000 Generation of a beam with a dark focus surrounded by regions of higher intensity: the optical bottle beam Opt. Lett. 25 191-3

Barnes W L et al 2003 Surface plasmon subwavelength optics Nature 424 824-30

Bazhenov V Y et al 1990 Laser-beams with screw dislocations in their wave-fronts Jetp Lett. 52 429-31

Beijersbergen M W et al 1994 Helical-wave-front laser-beams produced with a spiral phaseplate Opt. Commun. 112 321-7

Berry M V 1984 Quantal phase-factors accompanying adiabatic changes Proc of the Royal Society of London Series a-Mathematical Physical and Engineering Sciences vol 392 $45-57$

Blanchard R et al 2011 Off-axis and multi-directional plasmonic lenses 2011 Conf. on Lasers and Electro-Optics (CLEO) (Batimore, $M A$ )

Bomzon Z et al 2002 Space-variant Pancharatnam-Berry phase optical elements with computer-generated subwavelength gratings Opt. Lett. 27 1141-3

Brown B R and Lohmann A W 1966 Complex spatial filtering with binary masks Appl. Opt. 5 967-9

Brown B R and Lohmann A W 1969 Computer-generated binary holograms IBM J. Res. Dev. 13 160-8

Chen W T et al 2014 High-efficiency broadband meta-hologram with polarization-controlled dual images Nano Lett. 14225

Chen Y H et al 2011 Surface wave holography on designing subwavelength metallic structures Opt. Express 19 23908-20

Coullet P et al 1989 Optical vortices Opt. Commun. 73 403-8

Cowan J J 1972 Surface plasmon resonance effect in holography Bull. Am. Phys. Soc. 17133

Decker M, Staude I, Falkner M, Dominguez J, Neshev D N, Brener I, Pertsch T and Kivshar Y S 2014 High-efficiency light-wave control with all dielectric optical Huygens' metasurfaces arXiv: 1405.5038
Dolev I, Epstein I and Arie I 2012 Surface-plasmon holographic beam shaping Phys. Rev. Lett. 109203903

Dorn R et al 2003 Sharper focus for a radially polarized light beam Phys. Rev. Lett. 91233901

Ebbesen T W et al 1998 Extraordinary optical transmission through sub-wavelength hole arrays Nature 391 667-9

Gabor D 1948 A new microscopic principle Nature 161777

Garcia-Ortiz C E et al 2013 Generation of diffraction-free plasmonic beams with 1D Bessel profiles Opt. Lett. 38 905-7

Garcia-Vidal F J et al 2003 Focusing light with a single subwavelength aperture flanked by surface corrugations Appl. Phys. Lett. 83 4500-2

Genevet P et al 2010 Bistable and addressable localized vortices in semiconductor lasers Phys. Rev. Lett. 104223902

Genevet P et al 2012 Holographic detection of the orbital angular momentum of light with plasmonic photodiodes Nat. Commun. 31278

Genevet P et al 2012 Ultra-thin plasmonic optical vortex plate based on phase discontinuities Appl. Phys. Lett. 100013101

Genevet P et al 2013 Generation of 2D plasmonic bottle beams Opt. Express 21 10295-300

Genevet P and Capasso F 2014 Flat optics: wavefronts control with Huygens' interfaces IEEE Photon. J. 60700404

Ghadyani $\mathrm{Z}$ et al 2011 Concentric ring metal grating for generating radially polarized light Appl. Opt. 50 2451-7

Gibson G et al 2004 Free-space information transfer using light beams carrying orbital angular momentum Opt. Express $125448-56$

Golub M A et al 1996 Experimental investigation of a multibeam holographic optical element matched to Gauss-Laguerre modes Kvantovaya Elektronika 23 188-90

Goodman J W 1996 Introduction to Fourier Optics 2nd edn (New York: McGraw-Hill)

Gori F et al 1987 Bessel-Gauss beams Opt. Commun. 64 491-5

Grosjean T et al 2008 Linear to radial polarization conversion in the $\mathrm{THz}$ domain using a passive system Opt. Express 16 18895-909

Hall D G 1996 Vector-beam solutions of Maxwell's wave equation Opt. Lett. 21 9-11

Han Z H and Bozhevolnyi S I 2013 Radiation guiding with surface plasmon polaritons Rep. Prog. Phys. 76016402

$\mathrm{He} \mathrm{H}$ et al 1995 Direct observation of transfer of angularmomentum to absorptive particles from a laser-beam with a phase singularity Phys. Rev. Lett. 75 826-9

Heckenberg N R et al 1992 Generation of optical-phase singularities by computer-generated holograms Opt. Lett. 17 221-3

Heitmann D 1981 Calculation and experimental verification of 2D focusing grating couplers IEEE J. Quantum Electron. 1257-63

Hell S W 2007 Far-field optical nanoscopy Science 316 1153-8

Huang L L et al 2013 3D optical holography using a plasmonic metasurface Nat. Commun. 42808

Ishi T et al 2005 Si nano-photodiode with a surface plasmon antenna Japan. J. Appl. Phys. Part 2 Lett. Express Lett. 44 L364-6

Itai Epstein A A 2014 Shaping plasmonic light beams with nearfield plasmonic holograms JOSA B.

Juan M L et al 2011 Plasmon nano-optical tweezers Nat. Photon. $5349-56$

Karimi E et al 2007 Hypergeometric-gaussian modes Opt. Lett. 32 3053-5

Kawauchi $\mathrm{H}$ et al 2007 Calculation of optical trapping forces on a dielectric sphere in the ray optics regime produced by a radially polarized laser beam $O p t$. Lett. 32 1839-41

Kim S et al 2007 Off-axis directional beaming of optical field diffracted by a single subwavelength metal slit with asymmetric dielectric surface gratings Appl. Phys. Lett. 9051113 
Klein A E et al 2012 Controlling plasmonic hot spots by interfering Airy beams Opt. Lett. 37 3402-4

Kraus M et al 2010 Microdrilling in steel using ultrashort pulsed laser beams with radial and azimuthal polarization $\mathrm{Opt}$. Express 18 22305-13

Lalouat L et al 2011 Imaging photoexcited optical modes in photonic-crystal cavities with a near-field probe Phys. Rev. B 83201103

Larouche $\mathrm{S}$ et al 2012 Infrared metamaterial phase holograms Nat. Mater. 11 450-4

Lavery M P et al 2012 Refractive elements for the measurement of the orbital angular momentum of a single photon Opt. Express $202110-5$

Leach J et al 2002 Measuring the orbital angular momentum of a single photon Phys. Rev. Lett. 88257901

Lee B et al 2010 The use of plasmonics in light beaming and focusing Prog. Quantum Electron. 34 47-87

Lee W H 1979 Binary computer-generated holograms Appl. Opt. 18 3661-9

Leith E N and Upatniek J 1962 Reconstructed wavefronts and communication theory J. Opt. Soc. Am. 52 1123-8

Levy U et al 2004 Design, fabrication and characterization of subwavelength computer-generated holograms for spot array generation Opt. Express 12 5345-55

Levy U et al 2005 Near-infrared demonstration of computergenerated holograms implemented by using subwavelength gratings with space-variant orientation Opt. Lett. 30 2089-91

Lezec H J et al 2002 Beaming light from a subwavelength aperture Science 297 820-2

Li L et al 2013 Collimated plasmon beam: nondiffracting versus linearly focused Phys. Rev. Lett. 110046807

Lin J et al 2012 Cosine-Gauss plasmon beam: a localized longrange nondiffracting surface wave Phys. Rev. Lett. 109093904

Lin J et al 2013 Nanostructured holograms for broadband manipulation of vector beams Nano Lett. 13 4269-74

Lin J et al 2013 Polarization-controlled tunable directional coupling of surface plasmon polaritons Science 340 331-4

Liu Z W et al 2005 Focusing surface plasmons with a plasmonic lens Nano Lett. 5 1726-9

Lohmann A W and Paris D P 1967 Binary fraunhofer holograms generated by computer Appl. Opt. 6 1739-48

Lopez-Tejeira F et al 2007 Efficient unidirectional nanoslit couplers for surface plasmons Nat. Phys. 3 324-8

Lou K et al 2012 2D microstructures induced by femtosecond vector light fields on silicon Opt. Express 20 120-7

Maier S 2007 Plasmonics: Fundamentals and Applications (New York: Springer)

Mair A et al 2001 Entanglement of the orbital angular momentum states of photons Nature 412 313-6

Martín-Moreno L, García-Vidal F J, Lezec H J, Degiron A and Ebbesen T W 2003 Theory of highly directional emission from a single subwavelength aperture surrounded by surface corrugations Phys. Rev. Lett. 90167401

Maruo S et al 1997 Evanescent-wave holography: by use of surfaceplasmon resonance Appl. Opt. 36 2343-6

McGloin D et al 2003 3D arrays of optical bottle beams Opt. Commun. 225 215-22

Minovich A et al 2011 Generation and near-field imaging of airy surface plasmons Phys. Rev. Lett. 107116802

Montelongo Y et al 2014 Polarization switchable diffraction based on subwavelength plasmonic nanoantennas Nano Lett. $14294-8$

Monticone F et al 2013 Full control of nanoscale optical transmission with a composite metascreen Phys. Rev. Lett. 110201305

Ni X J et al 2012 Broadband light bending with plasmonic nanoantennas Science 335427

Ni X J et al 2013 Metasurface holograms for visible light Nat. Commun. 42807
Niziev V G et al 2006 Generation of inhomogeneously polarized laser beams by use of a Sagnac interferometer Appl. Opt. 45 8393-9

Niziev V G and Nesterov A V 1999 Influence of beam polarization on laser cutting efficiency J. Phys. D: Appl. Phys. 32 1455-61

Novotny L and Hecht B 2006 Principles of Nano-Optics (Cambridge, UK: Cambridge University Press)

Ozaki M et al 2011 Surface-plasmon holography with white-light illumination Science 332 218-20

Padgett M and Bowman R 2011 Tweezers with a twist Nat. Photon. $5343-8$

Pancharatnam S 1956 Generalized theory of interference, and its applications Proc. Indian Acad. Sci. A 44247

Pfeiffer C and Grbic A 2013 Metamaterial Huygens' surfaces: tailoring wave fronts with reflectionless sheets Phys. Rev. Lett. 110197401

Pohl D 1972 Operation of a Ruby-Laser in purely transverse electric mode Te01 Appl. Phys. Lett. 20266

Pors A et al 2013 Broadband focusing flat mirrors based on plasmonic gradient metasurfaces Nano Lett. 13 829-34

Regan C J et al 2012 2D Bessel-like surface plasmon-polariton beams J. Appl. Phys. 112103107

Righini M et al 2007 Parallel and selective trapping in a patterned plasmonic landscape Nat. Phys. 3 477-80

Salandrino A and Christodoulides D N 2010 Airy plasmon: a nondiffracting surface wave Opt. Lett. 35 2082-4

Schuller J A et al 2010 Plasmonics for extreme light concentration and manipulation Nat. Mater. 9 193-204

Shalaev V M and Kawata S 2007 Nanophotonics with Surface Plasmons. Advances in Nano-Optics and Nano-Photonics (Amsterdam: Elsevier)

Sheppard C J R and Choudhury A 2004 Annular pupils, radial polarization, and superresolution Appl. Opt. 43 4322-7

Siviloglou G A et al 2007 Observation of accelerating airy beams Phys. Rev. Lett. 99213901

Siviloglou G A and Christodoulides D N 2007 Accelerating finite energy Airy beams Opt. Lett. 32 979-81

Su T et al 2012 Demonstration of free space coherent optical communication using integrated silicon photonics orbital angular momentum devices Opt. Express 209396

Tetienne J P et al 2011 Dipolar modeling and experimental demonstration of multi-beam plasmonic collimators New J. Phys. 13053057

Tidwell S C et al 1990 Generating radially polarized beams interferometrically Appl. Opt. 29 2234-9

Torres J P and Torner L 2011 Twisted Photons: Applications of Light with Orbital Angular Momentum (Bristol: Wiley-VCH)

Tricoles G 1987 Computer generated holograms-An historical review Appl. Opt. 26 4351-60

Volpe G and Petrov D 2004 Generation of cylindrical vector beams with few-mode fibers excited by Laguerre-Gaussian beams Opt. Commun. 237 89-95

Walther B et al 2012 Spatial and spectral light shaping with metamaterials Adv. Mater. 24 6300-4

Wang G P et al 2001 Holography with surface-plasmon-coupled waveguide modes Appl. Opt. 40 3649-53

Wang H F et al 2008 Creation of a needle of longitudinally polarized light in vacuum using binary optics Nat. Photon. 2 501-5

Wang $\mathbf{J}$ et al 2012 Terabit free-space data transmission employing orbital angular momentum multiplexing Nat. Photon. 6 488-96

Wang K et al 2011 Trapping and rotating nanoparticles using a plasmonic nano-tweezer with an integrated heat sink Nat. Commun. 2469

Wang K and Crozier K B 2012 Plasmonic trapping with a gold nanopillar ChemPhysChem 13 2639-48

Weiner J 2009 The physics of light transmission through subwavelength apertures and aperture arrays Rep. Prog. Phys. 72064401 
Yelin D et al 2004 Generating an adjustable 3D dark focus Opt. Lett. 29 661-3

Yu L B et al 2005 Physical origin of directional beaming emitted from a subwavelength slit Phys. Rev. B 71041405

Yu N et al 2010 Plasmonics for laser beam shaping IEEE Trans. Nanotechnol. 911

Yu N F et al 2008 Small-divergence semiconductor lasers by plasmonic collimation Nat. Photon. 2 564-70

Yu N F et al 2011 Light propagation with phase discontinuities: generalized laws of reflection and refraction Science 334 333-7

Yu N F et al 2012 A broadband, background-free quarter-wave plate based on plasmonic metasurfaces Nano Lett. 12 6328-33

Yu N F et al 2013 Flat optics: controlling wavefronts with optical antenna metasurfaces IEEE J. Sel. Top. Quantum Electron. 194700423
Yu N F and Capasso F 2014 Flat optics with designer metasurfaces Nat. Mater. 13 139-50

Zhang P et al 2011 Plasmonic Airy beams with dynamically controlled trajectories Opt. Lett. 36 3191-3

Zhan Q W 2004 Trapping metallic Rayleigh particles with radial polarization Opt. Express 12 3377-82

Zhan Q W 2009 Cylindrical vector beams: from mathematical concepts to applications Adv. Opt. Photon. 1 1-57

Zhan Q W and Leger J R 2002 Interferometric measurement of the geometric phase in space-variant polarization manipulations Opt. Commun. 213 241-5

Zhao Y Q et al 2005 Creation of a 3D optical chain for controllable particle delivery Opt. Lett. $30848-50$

Zhou F et al 2013 Plasmonic holographic imaging with V-shaped nanoantenna array Opt. Express 21 4348-54 